\title{
Performance Analysis of General Tracking Algorithms
}

\author{
Lei Guo \\ Institute of Systems Science, Chinese Academy of Sciences \\ Beijing, 100080, China \\ and \\ Lennart Ljung \\ Department of Electrical Engineering, Linköping University \\ S-581 83 Linköping, Sweden
}

\begin{abstract}
A general family of tracking algorithms for linear regression models is studied. It includes the familiar LMS (gradient approach), RLS (recursive least squares) and KF (Kalman filter) based estimators. The exact expressions for the quality of the obtained estimates are complicated. Approximate, and easy-to-use, expressions for the covariance matrix of the parameter tracking error are developed. These are applicable over whole time interval, including the transient and the approximation error can be explicitly calculated.
\end{abstract}

\section{INTRODUCTION}

Tracking is the key factor in adaptive algorithms of all kinds. We shall in this contribution study the special case where the underlying model is a linear regression, i.e., the observations are related by

$$
y_{k}=\varphi_{k}^{\tau} \theta_{k}+v_{k} \quad, \quad k \geq 0 .
$$

Here $y_{k}$ is an observation made at time $k$, and $\varphi_{k}$ is a $d$ dimensional vector, that is known at time $k, v_{k}$ represents a disturbance and the parameter vector $\theta_{k}$ describes how the components of $\varphi_{k}$ relate to the observation $y_{k}$. It is the objective to estimate the vector $\theta_{k}$ from measurements $\left\{y_{t}, \varphi_{t}, t \leq k\right\}$.

Many technical problem formulations fit the structure (1) by choosing $\varphi_{k}$ and $y_{k}$ appropriately. See, among many references, for example, [15] and [22].

In order to come up with good algorithms for estimating $\theta_{k}$, it is natural to introduce some assumptions about the time-variation of this parameter vector. In general we may write

$$
\theta_{k}=\theta_{k-1}+\gamma w_{k}
$$

where $\gamma$ is a scaling constant and $w_{k}$ is an as yet undefined variable.

The tracking algorithms will provide us with an estimate

$$
\hat{\theta}_{k}=\hat{\theta}_{k}\left(y^{k}, \varphi^{k}, \theta^{k}\right)
$$

where superscript denotes the whole time history: $y^{k}=$ $\left\{y_{0}, y_{1}, \ldots y_{k}\right\}$, etc.

Supported by the National Natural Science Foundation of China Supported by the Swedish Research Council for Engineering Sciences (TFR)
A prime question concerns of course the quality of such an estimate. We shall evaluate the quality in terms of the covariance matrix of the tracking error

$$
\tilde{\theta}_{k}=\theta_{k}-\hat{\theta}_{k}
$$

This covariance matrix will be denoted by

$$
\Pi_{k}^{0}=E\left[\widetilde{\theta}_{k} \widetilde{\theta}_{k}^{\tau}\right]
$$

where expectation will be taken over all relevant stochastic variables. A precise definition will be given later.

An exact expression for $\Pi_{k}^{0}$ will be very complicated except in some trivial cases - and it will not be possible to derive it explicitly in closed form. However, the practical importance of having good tracking algorithms and estimates of their quality still makes it vital to be able to work with $\Pi_{k}^{0}$.

For that reason, there is a quite substantial literature on the problem of how to approximate $\Pi_{k}^{0}$ with expressions $\Pi_{k}$ that are simple to to work with. This literature is - partly - surveyed in [2], [1], [12], and [20].

The current paper has the ambition to give a general result that subsumes and extends most of the earlier results.

\section{Example 1.1 A Preview Example.}

Consider the model (1)-(2) under the assumptions that

a). $\varphi_{k}$ and $\theta_{k}$ are scalars;

b). $\left\{\varphi_{k}\right\},\left\{v_{k}\right\}$ and $\left\{w_{k}\right\}$ are independent sequences of independent random variables with zero mean values and variances $R_{\varphi}, R_{v}$ and $Q_{w}$, respectively.

c). The fourth moment of $\varphi_{k}$ is $R_{4}$.

Assume also that the estimate $\hat{\theta}_{k}$ is computed by the simple LMS algorithm

$$
\hat{\theta}_{k+1}=\hat{\theta}_{k}+\mu \varphi_{k}\left(y_{k}-\varphi_{k} \hat{\theta}_{k}\right) .
$$

This case is one - essentially the only one - where a simple exact expression for $\Pi_{k}^{0}$ can be calculated. Straightforward calculations give

$$
\widetilde{\theta}_{k+1}=\left(1-\mu \varphi_{k}^{2}\right) \widetilde{\theta}_{k}-\mu \varphi_{k} v_{k}+\gamma w_{k+1} .
$$

Squaring and taking expectations gives

$$
\Pi_{k+1}^{0}=\left(1-2 \mu R_{\varphi}+\mu^{2} R_{4}\right) \Pi_{k}^{0}+\mu^{2} R_{\varphi} R_{v}+\gamma^{2} Q_{w} .
$$


This is a linear time-invariant difference equation for $\Pi_{k}^{0}$, and can be explicitly solved. In particular, if

$$
\left|1-2 \mu R_{\varphi}+\mu^{2} R_{4}\right|<1,
$$

the solution of (8) will converge to $\Pi^{*}$ with

$$
\Pi^{*}=\frac{1}{1-\mu R_{4} /\left(2 R_{\varphi}\right)} \Pi, \quad \Pi=\frac{1}{2 R_{\varphi}}\left[\mu R_{\varphi} R_{v}+\frac{\gamma^{2}}{\mu} Q_{w}\right]
$$

Simple manipulations then give

$$
\left|\Pi^{*}-\Pi\right| \leq \sigma(\mu) \Pi, \quad \sigma(\mu)=\left[\frac{R_{4} /\left(2 R_{\varphi}\right)}{1-\mu R_{4} /\left(2 R_{\varphi}\right)}\right] \mu .
$$

Thus, $\Pi^{*}$ can be well approximated by $\Pi$ for small $\mu$, since $\sigma(\mu) \rightarrow 0$ as $\mu \rightarrow 0$.

Now, this example was particularly easy, primarily because of the assumed independence among $\left\{\varphi_{k}, v_{k}, w_{k}\right\}$ which makes $\varphi_{k}$ and $\widetilde{\theta}_{k}$ independent.

In more general cases we have to deal with dependence among $\left\{\varphi_{k}\right\}$, and that is actually at the root of the problem. Generally speaking, if $\left\{\varphi_{k}\right\}$ are weakly dependent, so should $\hat{\theta}_{k}$ and $\varphi_{k}$ be, provided that

$\hat{\theta}_{k}$ in (3) depends to a small extent on the "latest" $\varphi_{k}$, i.e. if the adaptation rate ( $\mu$ in the example) is small and the error equation ((7) in the example) is stable.

The extra term caused by the dependence in the equation corresponding to (8) in the example should then have negligible influence. Indeed, it is the purpose of this contribution to establish this for a fairly general family of tracking algorithms. Despite the simple idea, it turns out to be surprisingly technically difficult to prove. This paper could be said to make the end of a series of results on performance analysis, starting with Theorem 1 in [12] and then followed by [14],[13] and [10]. There are many related, relevant results using other approaches. We may point to [20], [2], [5], [6], [4], [16], [3] [18], and to the references in these books and papers.

The bottom line of the analysis is a result of the character

$$
\left\|E\left[\widetilde{\theta}_{k} \widetilde{\theta}_{k}^{\tau}\right]-\Pi_{k}\right\| \leq \sigma(\mu)\left\|\Pi_{k}\right\|,
$$

where $\sigma(\mu) \rightarrow 0$ as $\mu \rightarrow 0$, and $\mu$ is a measure of the adaptation rate in the algorithm, $\Pi_{k}$ obeys a simple linear, deterministic, difference equation (like (8) without the term $\left.\mu^{2} R_{4}\right)$.

The point with a result of the character (10) is, clearly, that we can arbitrarily well approximate the actual tracking error covariance matrix with a simple expression that can be easily evaluated and analyzed. The essence of this paper does not lie in the expression for $\Pi_{k}$ itself - it is not difficult to conjecture that such an approximation should be reasonable. Our contribution is rather to establish the connection in the explicit fashion (10) for a wide family of the most common tracking algorithms. One important step in achieving such results is to first establish that the underlying algorithm is exponentially stable. This is a major problem in itself, and a companion paper [9] is devoted to this step, for the same family of algorithms.
The paper is organized as follows. In Section 2 the tracking algorithms are briefly described. Section 3 gives the main result: That (10) holds under the same general conditions for all algorithms in the family. There we also briefly discuss the practical consequences of the result. In the following section, a more general theorem is presented, which is the basis for the analysis. This theorem is more general, and uses weaker but less explicit conditions. The proof of the main result is then given in Section 5, by showing that the general theorem can be applied to our family of algorithms. Notice that this analysis is of independent interest in that for each individual algorithm, the conditions can be somewhat weakened in different ways.

\section{The Family of Tracking Algorithms}

We shall consider the general adaptation algorithm

$$
\hat{\theta}_{k+1}=\hat{\theta}_{k}+\mu L_{k}\left(y_{k}-\varphi_{k}^{\tau} \hat{\theta}_{k}\right), \quad \mu \in(0,1),
$$

where the gain $L_{k}$ is chosen in some different ways:

\section{Case 1: Least Mean Squares (LMS):}

$$
L_{k}=\varphi_{k}
$$

This is a standard algorithm, [21],[22], and has been used in numerous adaptive signal processing applications.

\section{Case 2 : Recursive Least Squares(RLS):}

$$
\begin{aligned}
L_{k} & =P_{k} \varphi_{k} \\
P_{k} & =\frac{1}{1-\mu}\left\{P_{k-1}-\mu \frac{P_{k-1} \varphi_{k} \varphi_{k}^{\tau} P_{k-1}}{1-\mu+\mu \varphi_{k}^{\tau} P_{k-1} \varphi_{k}}\right\} \\
P_{0}>0 &
\end{aligned}
$$

This gives an estimate $\hat{\theta}_{k}$ that minimizes

$$
\sum_{t=1}^{k}(1-\mu)^{k-t}\left(y_{t}-\varphi_{t}^{\tau} \theta\right)^{2}
$$

where $(1-\mu)$ is the "forgetting factor".

Case 3: Kalman Filter (KF) Based Algorithm:

$$
\begin{aligned}
L_{k}= & \frac{P_{k-1} \varphi_{k}}{R+\mu \varphi_{k}^{\tau} P_{k-1} \varphi_{k}} \\
P_{k}= & P_{k-1}-\frac{\mu P_{k-1} \varphi_{k} \varphi_{k}^{\tau} P_{k-1}}{R+\mu \varphi_{k}^{\tau} P_{k-1} \varphi_{k}}+\mu Q, \\
& (R>0, Q>0)
\end{aligned}
$$

Here $R$ is a positive number and $Q$ is a positive definite matrix. The choice of $L_{k}$ corresponds to a Kalman filter state estimation for (1)-(2), and is optimal in the a posteriori mean square sense if $v_{k}$ and $w_{k}$ are Gaussian white noises with covariance matrices $R$ and $Q$, respectively, and if $\mu$ is chosen as $\gamma$ in (2).

If $\left\{\varphi_{k}, y_{k}, \theta_{k}\right\}$ obey (1) - (2) and $\hat{\theta}_{k}$ is found using (11) we can write the estimation error $\widetilde{\theta}_{k}$ as

$$
\widetilde{\theta}_{k+1}=\left(I-\mu F_{k}\right) \widetilde{\theta}_{k}-\mu L_{k} v_{k}+\gamma w_{k+1}, \quad F_{k}=L_{k} \varphi_{k}^{\tau}
$$


This is a purely algebraic consequence of (1) - (2) and (11), and holds for whatever sequences $v_{k}$ and $w_{k}$.

If we introduce stochastic assumptions about $\left\{v_{k}\right\}$ and $\left\{w_{k}\right\}$, we can use (19) to express the covariance matrix $E\left[\widetilde{\theta}_{k+1} \widetilde{\theta}_{k+1}^{\tau}\right]$. That will however be quite complex, primarily due to the dependence between $\left\{L_{k}, \varphi_{k}, \widetilde{\theta}_{k}\right\}$. The basic approximating expression will instead be based on the following expression

$\Pi_{k+1}=\left(I-\mu G_{k}\right) \Pi_{k}\left(I-\mu G_{k}\right)^{\tau}+\mu^{2} R_{v}(k) M_{k}+\gamma^{2} Q_{w}(k+1)$

where $G_{k}=E F_{k}, M_{k}=E L_{k} L_{k}^{\tau}, R_{v}(k)=E v_{k}^{2}$ and $Q_{w}(k)=E w_{k} w_{k}^{\tau}$. As follows from Example 1.1, this would be the correct expression for the covariance matrix of $\widetilde{\theta}_{k+1}$, if $v_{k}$ and $w_{k}$ were white noises and $L_{k} \varphi_{k}^{\tau}$ was independent of $\widetilde{\theta}_{k}$, and if a term of size $\mu^{2} \Pi_{k}$ was neglected.

Indeed, we shall prove that (20) provides a good approximation of the true covariance matrix in the sense that (10) holds. Note that $\Pi_{k}$ obeys a simple linear difference equation, and can easily be calculated and examined.

\section{The Main Result}

\section{A. The Assumptions}

We shall now consider the algorithm (11) with either of the three choices of the gain $L_{k}$, discussed in the previous section. For the analysis we shall impose some conditions on the involved variables. These are of the following character.

C1. The regressors $\left\{\varphi_{k}\right\}$ span the regressor space (in order to ensure that the whole parameter vector $\theta$ can be estimated);

C2. The dependence between the regressors $\varphi_{k}$ and $\left(\varphi_{i}, v_{i-1}, w_{i}\right)$ decays to zero as the time distance $(k-i)$ tends to infinity;

C3. The measured error $v_{k}$ and the parameter drift $w_{k}$ are of white noise character.

In more exact terms, the three assumptions take the following form:

P1. Let $S_{t}=E\left[\varphi_{t} \varphi_{t}^{\tau}\right]$, assume that there exist constants $h>0$ and $\delta>0$ such that

$$
\sum_{t=k+1}^{k+h} S_{t} \geq \delta I \quad, \quad \forall k
$$

P2. Let $\mathcal{G}_{k}=\sigma\left\{\varphi_{k}\right\}, \mathcal{F}_{k}=\sigma\left\{\varphi_{i}, v_{i-1}, w_{i}, i \leq k\right\}$. Assume that $\left\{\varphi_{k}\right\}$ is weakly dependent ( $\phi$-mixing) in the sense that there is a function $\phi(m)$ with $\phi(m) \rightarrow 0$, as $m \rightarrow \infty$, such that

$$
\sup _{A \in \mathcal{G}_{k+m}, B \in \mathcal{F}_{k}}|P(A \mid B)-P(A)| \leq \phi(m) \quad \forall k, \forall m .
$$

Also, assume that there is a constant $c_{\varphi}>0$ such that $\left\|\varphi_{k}\right\| \leq c_{\varphi}$ a.s., $\forall k$.
P3. Let $\mathcal{F}_{k}$ be the $\sigma$-algebra defined in P2, assume that

$$
\begin{aligned}
& E\left[v_{k} \mid \mathcal{F}_{k}\right]=0, \quad E\left[w_{k+1} \mid \mathcal{F}_{k}\right]=E\left[w_{k+1} v_{k} \mid \mathcal{F}_{k}\right]=0 \\
& E\left[v_{k}^{2} \mid \mathcal{F}_{k}\right]=R_{v}(k), \quad E\left[w_{k} w_{k}^{\tau}\right]=Q_{w}(k) \\
& \sup _{k}\left\{E\left[\left|v_{k}\right|^{r} \mid \mathcal{F}_{k}\right]+E\left\|w_{k}\right\|^{r}\right\} \leq M, \\
& \text { for some } \quad r>2, M>0 .
\end{aligned}
$$

\section{B. The Result}

Now, let $\Pi_{k}$ be defined by the following linear, deterministic difference equation:

$$
\begin{aligned}
\Pi_{k+1}= & \left(I-\mu R_{k} S_{k}\right) \Pi_{k}\left(I-\mu R_{k} S_{k}\right)^{\tau} \\
& +\mu^{2} R_{v}(k) R_{k} S_{k} R_{k}+\gamma^{2} Q_{w}(k+1)
\end{aligned}
$$

where $S_{k}=E\left[\varphi_{k} \varphi_{k}^{\tau}\right]$, and $R_{k}$ is defined as follows:

\section{LMS-case}

$$
R_{k}=I
$$

\section{RLS-case}

$$
R_{k}=R_{k-1}-\mu R_{k-1} S_{k} R_{k-1}+\mu R_{k-1}, \quad\left(R_{0}=P_{0}\right)
$$

\section{KF-case}

$$
R_{k}=R_{k-1}-\mu R_{k-1} S_{k} R_{k-1}+\mu Q / R, \quad\left(R_{0}=P_{0} / R\right)
$$

We then have the following main result.

Theorem 3.1 Consider any of the three basic algorithms in Section 2. Assume that P1, P2 and P3 hold. Let $\Pi_{k}$ be defined as above. Then $\forall \mu \in\left(0, \mu^{*}\right), \forall k \geq 1$

$$
\left\|E\left[\widetilde{\theta}_{k} \widetilde{\theta}_{k}^{\tau}\right]-\Pi_{k}\right\| \leq c \sigma(\mu)\left[\mu+\frac{\gamma^{2}}{\mu}+(1-\alpha \mu)^{k}\right]
$$

where $\sigma(\mu) \rightarrow 0($ as $\mu \rightarrow 0)$, which is defined by

$$
\sigma(\mu) \triangleq \min _{m \geq 1}\{\sqrt{\mu} m+\phi(m)\}
$$

and $\phi(m)$ was defined in P2, and $\alpha \in(0,1), \mu^{*} \in(0,1), c>$ 0 are constants which may be computed using properties of $\left\{\varphi_{k}, v_{k}, w_{k}\right\}$.

The proof is given in Section 5. Let us now discuss the conditions used in the above theorem.

\section{The Degree of Approximation}

First of all, it is clear that the quantity $\sigma(\mu)$ plays an important role. The faster it tends to zero, the better approximation is obtained. The rate by which it tends to zero is according to $(27)$ a reflection of how fast $\phi(m)$ (that is, the dependence among the regressors) tends to zero as $m$ increases. For example, if the regressors are $m$-dependent, so that $\varphi_{k}$ and $\varphi_{\ell}$ are independent for $|k-\ell|>m$, then $\phi(n)=0$ for $n>m$ and $\sigma(\mu)$ will behave like $\sqrt{\mu}$. Also, if the dependence is exponentially decaying $\left(\phi(m) \approx C e^{-\alpha m}\right)$, then we can find that

$$
\sigma(\mu)<C \mu^{0.5-\delta}
$$

for arbitrarily small, positive $\delta$. This gives a good picture of typical decay rates of $\sigma$. 


\section{Persistence of Excitation: Condition P1}

Condition $\mathrm{P} 1$ is quite natural and weak, just requiring the regressor covariance matrix to add up to full rank over a given time span of arbitrary length. It has been known to be a necessary condition (in a certain sense) for boundedness of $E\left\|\widetilde{\theta}_{k}\right\|^{2}$ generated by LMS (cf. [8]), it is also known to be the minimum excitation condition needed for the stability analysis of RLS (cf. [10]).

\section{E. Boundedness and $\phi$-mixing of the regressors: Condition P2}

Condition P2 requires boundedness and $\phi$-mixing of the regressors. Although such conditions are standard ones in the literature (e.g. [11]), they can still be considered as restrictive. As seen in several of the results in Section 5, both $\phi$-mixing and boundedness can be weakened considerably when we deal with specific algorithms.

It may also be remarked that when $\left\{\varphi_{k}\right\}$ is unbounded, we can modify the algorithm and make Theorem 3.1 hold true: Introduce the normalized signal

$$
\left(\bar{y}_{k}, \bar{\varphi}_{k}, \bar{v}_{k}\right)=\frac{1}{\sqrt{1+\left\|\varphi_{k}\right\|^{2}}}\left(y_{k}, \varphi_{k}, v_{k}\right)
$$

Then we have from (1)

$$
\bar{y}_{k}=\theta_{k}^{\tau} \bar{\varphi}_{k}+\bar{v}_{k}
$$

Thus, $\left\{\theta_{k}\right\}$ may be estimated based on this normalized linear regression. In this case, Theorem 3.1 can be applied to this case if only $S_{k}$ and $R_{v}(k)$ in (22)-(25) are replaced by $E\left[\frac{\varphi_{k} \varphi_{k}^{\tau}}{1+\left\|\varphi_{k}\right\|^{2}}\right]$ and $E\left[\frac{1}{1+\left\|\varphi_{k}\right\|^{2}}\right] R_{v}(k)$, respectively.

\section{F. The Parameter Drift Model: Condition P3}

There are two things to mention around the Conditions P3. First, we note that the martingale difference property of $w_{k}$ essentially means that the true parameters, according to the model (2) are assumed to be a random walk. Although this model is quite standard, it has also been criticized as being too restrictive. We believe that a random walk model, in the context of slow adaptation (small $\mu$ ), captures the tracking behavior of the algorithm very well. This is, in a sense, a worst case analysis, since the future behavior of the model is unpredictable.

We may also note that time-varying covariances $Q_{w}(k)$ and $R_{v}(k)$ are allowed. Several of the special model drift cases described in [12] are therefore covered by P3. Other drift models, where the driving noise is colored, can be put into a similar Kalman filter framework. However, to cover also that case with our techniques requires more work.

Condition P3 also introduces assumptions about higher moments than 2. We remark that if we only assume that $\left\{v_{k}\right\}$ and $\left\{w_{k}\right\}$ are bounded in e.g. mean square sense, then upper bounds for the mean square tracking errors can be established (cf. [8] and [7]). The strengthened assumption in P3 allows us to obtain performance values much more accurate than upper bounds.

\section{G. The Practical Use of the Theorem}

The practical consequences of Theorem 3.1 is that a very simple algorithm, the linear, deterministic difference equation (22) will describe the tracking behavior. Now, this equation is quite easy to analyze. In fact, there is an extensive literature on such analysis, in particular for the special case of LMS. Among many references, we may refer to [12] for a survey of such results. In essence, all these results capture the dilemma between tracking error ( $\Pi$ is large because $\mu$ is small) and the noise sensitivity ( $\Pi$ is large because $\mu$ is large) and may point to the best compromises between these requirements.

For example, under weak stationarity of the regressors

$$
S_{k} \equiv S
$$

we find that $R_{k}$ will converge to $\tilde{R}$ as $k \rightarrow \infty$, where $\tilde{R}=I$ in the LMS-case, $\tilde{R}=S^{-1}$ in the RLS case and for the KF case we have to solve

$$
\tilde{R} S \tilde{R}=Q / R
$$

for $\tilde{R}$. Inserted into (22) this gives the following stationary values $\Pi$ for the tracking error covariance matrix (neglecting the term $\left.\mu^{2} \Pi\right)$ :

$$
\begin{gathered}
\text { LMS } S \Pi+\Pi S=\mu R_{v} S+\frac{\gamma^{2}}{\mu} Q_{w} \\
\text { RLS } \Pi=\frac{1}{2}\left[\mu R_{v} S^{-1}+\frac{\gamma^{2}}{\mu} Q_{w}\right] \\
\text { KF } \quad \tilde{R} S \Pi+\Pi(\tilde{R} S)^{\tau}=\mu R_{v} Q / R+\frac{\gamma^{2}}{\mu} Q_{w}
\end{gathered}
$$

Note, that if we have $Q=Q_{w}$ and $R=R_{v}$, then the latter equation can be solved as

$$
\Pi=\frac{R}{2}\left(\mu+\frac{\gamma^{2}}{\mu}\right) \tilde{R}
$$

From these expressions the trade-offs between tracking ability and noise sensitivity are clearly visible.

\section{A General Theorem}

In this section, we shall present a general theorem on performance of tracking algorithm (11) when the gain $L_{k}$ is not specified, from which our main result Theorem 3.1 will follow. The general theorem has weaker, but less explicit assumptions. From now on the treatment and discussion will be more technical. However, the main line of thought in the proofs follows the outline given after Example 1.1 in the Introduction.

\section{A. Notations}

The following notations will be used in the remainder of the paper. These notations are the same as in the companion paper [9]. 
a). The minimum and maximum eigenvalues of a matrix $X$ are denoted by $\lambda_{\min }(X)$ and $\lambda_{\max }(X)$, respectively, and

$$
\begin{aligned}
\|X\| & \triangleq\left\{\lambda_{\max }\left(X X^{\tau}\right)\right\}^{\frac{1}{2}} \\
\|X\|_{p} & \triangleq\left\{E\left(\|X\|^{p}\right)\right\}^{\frac{1}{p}}, \quad p \geq 1 .
\end{aligned}
$$

b). Let $x=\left\{x_{k}(\mu), k \geq 1\right\}$ be a random sequence parameterized by $\mu \in(0,1)$. Denote

$$
\mathcal{L}_{p}\left(\mu^{*}\right)=\left\{x: \quad \sup _{\mu \in\left(0, \mu^{*}\right]} \sup _{k \geq 1}\left\|x_{k}(\mu)\right\|_{p}<\infty\right\}
$$

c). Let $F=\left\{F_{k}(\mu)\right\}$ be any (square) matrix random process parameterized by $\mu \in(0,1)$. For any $p \geq 1, \quad \mu^{*} \in$ $(0,1)$, define

$\mathcal{S}_{p}\left(\mu^{*}\right)=\left\{F:\left\|\prod_{j=i+1}^{k}\left(I-\mu F_{k}(\mu)\right)\right\|_{p} \leq M(1-\mu \alpha)^{k-i}\right.$,

$\forall \mu \in\left(0, \mu^{*}\right], \forall k \geq i \geq 0, \quad$ for some $M>0$ and $\left.\alpha \in(0,1)\right\}$

similarly,

$\mathcal{S}\left(\mu^{*}\right)=\left\{F:\left\|\prod_{j=i+1}^{k}\left(I-\mu E\left[F_{k}(\mu)\right]\right)\right\| \leq M(1-\mu \alpha)^{k-i}\right.$,

$\forall \mu \in\left(0, \mu^{*}\right], \forall k \geq i \geq 0, \quad$ for some $M>0$, and $\left.\alpha \in(0,1)\right\}$

In what follows, it will be convenient to introduce the sets

$$
\mathcal{S}_{p} \triangleq \bigcup_{\mu^{*} \in(0,1)} \mathcal{S}_{p}\left(\mu^{*}\right), \quad \mathcal{S} \triangleq \bigcup_{\mu^{*} \in(0,1)} \mathcal{S}\left(\mu^{*}\right),
$$

We may call these stability sets. They are related to the stability of random equation (19) and deterministic equation (20), respectively. For simplicity, we shall sometimes suppress the parameter $(\mu)$ in $F_{k}(\mu)$, when there is no risk of confusion.

d). For scalar random sequences $a=\left(a_{k}, k \geq 0\right)$, we set

$$
\begin{gathered}
\mathcal{S}^{0}(\lambda)= \\
\left\{a: a_{k} \in[0,1], E \prod_{j=i+1}^{n}\left(1-a_{j}\right) \leq M \lambda^{k-i},\right. \\
\forall k \geq i \geq 0, \text { for some } \quad M>0\} .
\end{gathered}
$$

Also,

$$
\mathcal{S}^{0} \triangleq \bigcup_{\lambda \in(0,1)} \mathcal{S}^{0}(\lambda)
$$

e). Let $p \geq 1$ and let $x \triangleq\left\{x_{i}\right\}$ be any random process. Set

$\mathcal{M}_{p}=\left\{x:\left\|\sum_{i=m+1}^{m+n} x_{i}\right\|_{p} \leq C_{p} n^{\frac{1}{2}}, \forall n \geq 1, m \geq 0\right.$,

for some $C_{p}$ depending only on $p$ and $\left.x\right\}$.
As is known for example from [10], martingale difference sequence, $\phi$ - and $\alpha$-mixing sequences, and linear processes (a process generated from a white noise source via a linear filter with absolutely summable impulse response) are all in the set $\mathcal{M}_{p}$.

In particular, when $\left\{x_{i}\right\}$ is a martingale difference sequence, by the Burkholder inequality we have $(p>1)$

$$
\left\|\sum_{i=m+1}^{m+n} x_{i}\right\|_{p} \leq\left(B_{p} x_{p}^{*}\right) n^{\frac{1}{2}}, \quad \forall n \geq 1, m \geq 0
$$

where $x_{p}^{*} \triangleq \sup _{k}\left\|x_{k}\right\|_{p}$, and $B_{p}$ is a constant depending on $p$ only (cf. [11]). (This fact will be frequently used in the sequel without explanations).

f). Let $\left\{A_{k}\right\}$ be a matrix sequence, $b_{k} \geq 0, \forall k \geq 0$. Then by $A_{k}=O\left(b_{k}\right)$ we mean that there exists a constant $M<\infty$ such that

$$
\left\|A_{k}\right\| \leq M b_{k} \quad, \quad \forall k \geq 0
$$

The constant $M$ may be called the ordo-constant. Throughout the sequel, the ordo-constant does not depend on $\mu$, even if $\left\{A_{k}\right\}$ or $\left\{b_{k}\right\}$ does.

\section{B. Assumptions}

We will first show that given the exponential stability of the homogenous part of (19) and a certain weak dependence property of the adaptation gains, how the tracking performance can be analyzed, then we present more detailed discussions on such properties.

In the sequel, unless otherwise stated, $\mathcal{F}_{k}$ denotes the $\sigma$-algebra generated by $\left\{\varphi_{i}, w_{i}, v_{i-1}, i \leq k\right\}$, and $\left\{F_{k}\right\}$ is defined in (19).

To establish the general theorem, we need the following assumptions:

(A1). (Exponential stability) There are $\mu^{*} \in(0,1)$, and $p \geq 2$ such that

$$
\left\{F_{k}\right\} \in \mathcal{S}_{p}\left(\mu^{*}\right) \bigcap \mathcal{S}\left(\mu^{*}\right)
$$

(A2). (Weak dependence) There is a real number $q \geq 3$ together with a bounded function $\phi(m, \mu) \geq 0$, with

$$
\lim _{\substack{m \rightarrow \infty \\ \mu \rightarrow 0}} \phi(m, \mu)=0
$$

(taking first $m$ to infinity and then $\mu$ to zero) such that $\forall m, \forall k, \forall \mu \in\left(0, \mu^{*}\right]$

$$
\left\|E\left[F_{k} \mid \mathcal{F}_{k-m}\right]-E\left[F_{k}\right]\right\|_{q} \leq \phi(m, \mu)
$$

(A3). $L_{i} \in \mathcal{F}_{i}, \forall i \geq 1$, and there is $\mu^{*} \in(0,1)$ such that

$$
\left\{L_{i}\right\} \in \mathcal{L}_{r}\left(\mu^{*}\right), \quad\left\{F_{i}\right\} \in \mathcal{L}_{2 q}\left(\mu^{*}\right),
$$

with $r=\left(\frac{1}{2}-\frac{1}{p}-\frac{3}{2 q}\right)^{-1}$, and with $\mathrm{p}$ and $\mathrm{q}$ defined as in (A1) and (A2). 
(A4). For all $k \geq 1$ we have

$$
\begin{gathered}
E\left[v_{k} \mid \mathcal{F}_{k}\right]=0, \quad E\left[w_{k+1} \mid \mathcal{F}_{k}\right]=E\left[w_{k+1} v_{k} \mid \mathcal{F}_{k}\right]=0 \\
E\left[v_{k}^{2} \mid \mathcal{F}_{k}\right]=R_{v}(k), \quad E\left[w_{k+1} w_{k+1}^{\tau}\right]=Q_{w}(k+1) \\
E\left[\left|v_{k}\right|^{r} \mid \mathcal{F}_{k}\right]+E\left[\left\|w_{k+1}\right\|^{r}\right] \leq M<\infty \quad, \forall k \geq 1
\end{gathered}
$$

for deterministic quantities $R_{v}(k), Q_{w}(k+1)$ and $M$, where $r$ is defined as in (A3).

The key conditions are (A1) and (A2). In general, (A1) can be guaranteed by a certain type of stochastic persistence of excitation condition, which is studied in the companion paper [9]; while (A2) can be guaranteed by imposing a certain weak dependence condition on the regressor $\left\{\varphi_{i}\right\}$. More detailed discussions will be given later. At the moment, we just remark that if (A1) and (A2) hold for all $p \geq 1$ and all $q \geq 1$, then in (A3) and (A4), the number $r$ needs only to satisfy $r>2$.

\section{The General Theorem}

Now, recursively define a matrix sequence $\left\{\hat{\Pi}_{k}\right\}$ as follows:

$$
\begin{aligned}
\hat{\Pi}_{k+1}= & \left(I-\mu E\left[F_{k}\right]\right) \hat{\Pi}_{k}\left(I-\mu E\left[F_{k}\right]\right)^{\tau} \\
& +\mu^{2} R_{v}(k) E\left[L_{k} L_{k}^{\tau}\right]+\gamma^{2} Q_{w}(k+1),
\end{aligned}
$$

where $\hat{\Pi}_{0}=E\left[\widetilde{\theta}_{0} \widetilde{\theta}_{0}^{\tau}\right]$, and $R_{v}(k)$ and $Q_{w}(k+1)$ are defined in Assumption (A4). Note that this definition is very close to the definition of $\Pi_{k}$ in (22). We now have a result that is the "mother-theorem" of Theorem 3.1:

Theorem 4.1 Let Assumptions (A1)-(A4) hold. Let the tracking error $\widetilde{\theta}_{k}$ be defined by (11) (or (19)), and let $\hat{\Pi}_{k}$ defined by (32). Then $\forall \mu \in\left(0, \mu^{*}\right], \forall k \geq 1$ :

$$
\left\|E\left[\widetilde{\theta}_{k+1} \widetilde{\theta}_{k+1}^{\tau}\right]-\hat{\Pi}_{k+1}\right\| \leq c \sigma(\mu)\left[\mu+\frac{\gamma^{2}}{\mu}+(1-\alpha \mu)^{k}\right]
$$

where $c>0$ and $\alpha \in(0,1)$ are constants and $\sigma(\mu)$ is a function that tends to zero as $\mu$ tends to zero. It is defined by

$$
\sigma(\mu) \triangleq \min _{m \geq 1}\{\sqrt{\mu} m+\phi(m, \mu)\} .
$$

The proof is given in Appendix A.

Next, we show that under more conditions, the expression for $\hat{\Pi}_{k}$ in (32) can be further simplified.

Corollary 4.1. Under the conditions of Theorem 4.1, if $F_{k}=P_{k} \varphi_{k} \varphi_{k}^{\tau}$ with $\left\|\varphi_{k}\right\|_{2 t}=O(1),\left\|F_{k}\right\|_{t}=O(1)$, for some $t>1$, and if there are some function $\delta(\mu)$, tending to zero as $\mu$ tends to zero, and some deterministic sequence $\left\{R_{k}\right\}$ such that

$$
\left\|P_{k}-R_{k}\right\|_{s}=O(\delta(\mu)) \quad \forall k, \forall \mu \in\left(0, \mu^{*}\right], \quad s=\left(1-t^{-1}\right)^{-1}
$$

then we have $\left(\forall \mu \in\left(0, \mu^{*}\right], \forall k \geq 1\right)$

$$
\begin{aligned}
& \left\|E\left[\widetilde{\theta}_{k+1} \widetilde{\theta}_{k+1}^{\tau}\right]-\Pi_{k+1}\right\| \\
& \leq c[\sigma(\mu)+\delta(\mu)]\left[\mu+\frac{\gamma^{2}}{\mu}+(1-\alpha \mu)^{k}\right]
\end{aligned}
$$

for some constants $c>0$ and $\alpha \in(0,1)$, where $\Pi_{k}$ is recursively defined by

$$
\begin{aligned}
& \Pi_{k+1}=\left(I-\mu R_{k} S_{k}\right) \Pi_{k}\left(I-\mu R_{k} S_{k}\right)^{\tau}+ \\
& \mu^{2} R_{v}(k) R_{k} S_{k} R_{k}^{\tau}+\gamma^{2} Q_{w}(k+1),
\end{aligned}
$$

with $S_{k}=E\left[\varphi_{k} \varphi_{k}^{\tau}\right]$ and $\Pi_{0}=\hat{\Pi}_{0}$.

Proof. By Theorem 4.1, we need only to show that

$$
\left\|\hat{\Pi}_{k+1}-\Pi_{k+1}\right\|=O\left(\delta(\mu)\left[\mu+\frac{\gamma^{2}}{\mu}+(1-\alpha \mu)^{k}\right]\right),
$$

This can be derived by straightforward calculations based on the equations for $\hat{\Pi}_{k}$ and $\Pi_{k}$, and hence Corollary 4.1 is true.

Remark. If in Condition (A2),

$$
\phi(m, \mu)=O(\phi(m)+\delta(\mu)), \quad \delta(\mu)=\min _{m \geq 1}[\sqrt{\mu} m+\phi(m)],
$$

then $\sigma(\mu)$ defined in Theorem 4.1 satisfies $\sigma(\mu)=O(\delta(\mu))$. This will be the case for RLS and KF algorithms in Theorem 3.1, as can be seen from section V.

The following result also follows directly from Theorem 4.1 .

Corollary 4.2. If, in addition to the conditions of Theorem $4.1, R_{v}(k) \equiv R_{v}, Q_{w}(k) \equiv Q_{w}$, and there are $F, G$, and a function $\delta(\mu)$, tending to zero as $\mu$ tends to zero, such that $\forall \mu \in\left(0, \mu^{*}\right]$,

$$
\left\|E F_{k}-F\right\|+\left\|E\left(L_{k} L_{k}^{\tau}\right)-G\right\| \leq \delta(\mu), \quad \forall k
$$

then for some $\alpha \in(0,1)$ and for all $\mu \in\left(0, \mu^{*}\right], k \geq 1$,

$$
\begin{aligned}
& E\left[\widetilde{\theta}_{k+1} \widetilde{\theta}_{k+1}^{\tau}\right] \\
& =\Pi+O\left([\sigma(\mu)+\delta(\mu)]\left[\mu+\frac{\gamma^{2}}{\mu}\right]\right)+O\left((1-\alpha \mu)^{k}\right)(35)
\end{aligned}
$$

where $\Pi$ satisfies the following Lyapunov equation:

$$
F \Pi+\Pi F^{\tau}=\mu R_{v} G+\frac{\gamma^{2}}{\mu} Q_{w} .
$$

Now denote

$$
\bar{R}_{v}=R_{v} \int_{0}^{\infty} e^{-F t} G e^{-F^{\tau} t}, \quad \bar{Q}_{w}=\int_{0}^{\infty} e^{-F t} Q_{w} e^{-F^{\tau} t}
$$

the solution to the Lyapunov equation (36) can be expressed as

$$
\Pi=\mu \bar{R}_{v}+\frac{\gamma^{2}}{\mu} \bar{Q}_{w},
$$

in which there is a reminiscence of the results obtained in the simple example discussed in Section 1 (see, (9)). 
D. Discussion on the Assumptions

Now, let us discuss the key assumptions (A1) and (A2).

First, assumption (A1) has been studied in the companion paper [9], and here we only give some results concerning $\left\{F_{k}\right\} \in \mathcal{S}$, which will be used shortly in the next section.

Proposition 4.1. Let $\left\{G_{k}\right\}$ be a random matrix process, possibly dependent on $\mu$, with the property

$$
E\left\|G_{k}\right\| \leq \delta(\mu) \quad, \quad \text { for all small } \mu \text { and all } k
$$

where $\delta(\mu) \rightarrow 0$ as $\mu \rightarrow 0$.

Then $\left\{F_{k}\right\} \in \mathcal{S} \Longleftrightarrow\left\{F_{k}+G_{k}\right\} \in \mathcal{S}$.

Proof. Sufficiency: Recursively define $(\forall x:\|x\|=1)$

$$
x_{k+1}=\left(I-\mu E\left[F_{k}+G_{k}\right]\right) x_{k}, \quad \forall k \geq m, x_{m}=x,
$$

Then

$$
\begin{aligned}
x_{k+1}= & \left(I-\mu E\left(F_{k}\right)\right) x_{k}-\mu E\left(G_{k}\right) x_{k} \\
= & \prod_{i=m}^{n}\left[I-\mu E\left(F_{i}\right)\right] x_{m} \\
& -\sum_{i=m}^{n} \mu \prod_{j=i+1}^{n}\left[I-\mu E\left(F_{j}\right)\right] E\left(G_{i}\right) x_{i}
\end{aligned}
$$

Consequently, similar to the proof of Theorem 3.1 in [9], by the Gronwall inequality we have

$$
\begin{aligned}
\left\|x_{n+1}\right\| \leq & 2 M(1-\mu \alpha)^{n-m+1} \\
& \times\left\{1+\sum_{i=m}^{n} \prod_{j=i+1}^{n}\left(1+\mu E\left\|G_{j}\right\|\right) \cdot \mu E\left\|G_{i}\right\|\right\}
\end{aligned}
$$

From this and the condition (37), it is not difficult to convince oneself that $\left\{F_{k}+G_{k}\right\} \in \mathcal{S}$.

Necessity: by using the fact proved above, and noting that $F_{k}=\left(F_{k}+G_{k}\right)-G_{k}$, we know that $\left\{F_{k}\right\} \in \mathcal{S}$. This completes the proof.

The following useful result follows from Proposition 4.1 immediately.

Proposition 4.2. Let $F_{k}=P_{k} H_{k}$ and the following conditions be satisfied:

(i). $\left\{H_{k}\right\} \in \mathcal{L}_{t}\left(\mu^{*}\right), \quad \mu^{*} \in(0,1), t \geq 1$.

(ii). $\left\|P_{k}-\bar{P}_{k}\right\|_{s} \leq \delta(\mu),, \quad \forall \mu \in\left(0, \mu^{*}\right]$, where, $\delta(\mu) \rightarrow 0$ as $\mu \rightarrow 0, s=\left(1-t^{-1}\right)^{-1}$, and $\left\{\bar{P}_{k}\right\}$ is a deterministic process.

Then $\left\{F_{k}\right\} \in \mathcal{S} \Longleftrightarrow\left\{\bar{P}_{k} H_{k}\right\} \in \mathcal{S}$.

Proof. The result follows directly from Proposition 4.1, if we note that

$$
F_{k}=\bar{P}_{k} H_{k}+\left(P_{k}-\bar{P}_{k}\right) H_{k} .
$$

We now turn to discuss the weak dependence condition (A2).
Example 4.1 Let $\left\{\varphi_{i}\right\}$ satisfy $(21)$, and $L(\cdot): R^{d} \longrightarrow$ $R^{d \times d}$ be a real matrix function with $\|L(\varphi(k))\|_{q}=O(1)$, for some $1 \leq q \leq \infty$. Then we have the following inequality (c.f.[19]):

$$
\left\|E\left[L\left(\varphi_{k}\right) \mid \mathcal{F}_{k-m}\right]-E L\left(\varphi_{k}\right)\right\|_{q}=O\left([\phi(m)]^{1-\frac{1}{q}}\right) . \quad \forall k, m
$$

Hence, if $F_{k}=L\left(\varphi_{k}\right)$, then condition (A2) holds.

Note that when $\left\{\varphi_{k}\right\}$ satisfies condition P2 in Section 3, we have by taking $q=\infty$ in (38)

$$
\left\|E\left[\varphi_{k} \varphi_{k}^{\tau} \mid \mathcal{F}_{k-m}\right]-E \varphi_{k} \varphi_{k}^{\tau}\right\|_{\infty}=O(\phi(m)) .
$$

This fact will be used in the next section in the proof of Theorem 3.1.

Example 4.2 Let $\left\{\varphi_{k}\right\}$ be generated by

$$
\left\{\begin{array}{l}
x_{k}=A x_{k-1}+B \xi_{k}, \quad \text { (A stable). } \\
\varphi_{k}=C x_{k}+\xi_{k}
\end{array}\right.
$$

where $\left\{\xi_{j}, j \geq k+1\right\}$ and $\left\{v_{j-1}, w_{j}, j \leq k\right\}$ are independent, and $\left\{\xi_{j}\right\}$ is an independent sequence. Assume that

$$
\sup _{k} E\left\|\xi_{k}\right\|^{(b+1) q}<\infty, \quad \text { for some } \quad b \geq 0, q \geq 1 .
$$

Then for any function $L(\cdot): R^{d} \longrightarrow R^{d \times d}$, with

$$
\left\|L(x)-L\left(x^{\prime}\right)\right\| \leq M\left(\|x\|+\left\|x^{\prime}\right\|+1\right)^{b}\left\|x-x^{\prime}\right\|, \quad \forall x, x^{\prime},
$$

there is a constant $\lambda \in(0,1)$ such that (cf.[14]) $\forall m \geq 0 \forall k \geq$ 0

$$
\left\|E\left[L\left(\varphi_{k+m}\right) \mid \mathcal{F}_{k}\right]-E L\left(\varphi_{k+m}\right)\right\|_{q}=O\left(\lambda^{m}\right)
$$

Hence, if $F_{k}=L\left(\varphi_{k}\right)$, then again, condition (A2) holds.

following simple result will be useful in the sequel.

Proposition 4.3 Let $F_{k}=P_{k} L\left(\varphi_{k}\right)$, and the following two conditions hold:

(i). There is a bounded deterministic matrix sequence $\left\{\bar{P}_{k}\right\}$, and a function $\delta(\mu)$ tending to zero as $\mu$ tends to zero, such that

$$
\left\|P_{k}-\bar{P}_{k}\right\|_{s} \leq \delta(\mu), \quad \forall \mu \in\left(0, \mu^{*}\right], \quad \text { for some } \quad s>1 ;
$$

(ii). There is a number $r>1$ such that $\left\|L\left(\varphi_{k}\right)\right\|_{r}=$ $O(1)$, together with a function $\phi(m)$ tending to 0 as $m$ tends to infinity, such that

$$
\begin{gathered}
\left\|E\left[L\left(\varphi_{k+m}\right) \mid \mathcal{F}_{k}\right]-E L\left(\varphi_{k+m}\right)\right\|_{q} \leq \phi(m), \\
\forall k, \forall m, \quad\left(q=\left(r^{-1}+s^{-1}\right)^{-1}\right) .
\end{gathered}
$$

Then condition (A2) holds with $\phi(m, \mu)=O(\phi(m)+\delta(\mu))$.

Proof. The result follows directly from the following identity:

$$
\begin{aligned}
E[ & \left.F_{k+m} \mid \mathcal{F}_{k}\right]-E F_{k+m} \\
= & {\left[\left(P_{k+m}-\bar{P}_{k+m}\right) L\left(\varphi_{k+m}\right) \mid \mathcal{F}_{k}\right] } \\
& -E\left[\left(P_{k+m}-\bar{P}_{k+m}\right) L\left(\varphi_{k+m}\right)\right] \\
& +\bar{P}_{k+m}\left\{E\left[L\left(\varphi_{k+m}\right) \mid \mathcal{F}_{k}\right]-E L\left(\varphi_{k+m}\right)\right\} .
\end{aligned}
$$


V. Analysis of the Basic Algorithms

In this section, we shall show that, for the basic LMS, RLS and KF algorithms, conditions (A1)-(A3) of the previous section can be guaranteed by imposing some explicit (stochastic excitation and weak dependence) conditions on the regressors $\left\{\varphi_{k}\right\}$, and at the same time prove Theorem 3.1 .

\section{A. Analysis of $L M S$}

For the LMS defined by (11) - (12), let us introduce the following two kinds of weak dependence conditions:

L1). Condition P2 of Section 3 is satisfied but with the boundedness condition on $\left\{\varphi_{k}\right\}$ relaxed to the following : There exist positive constants $\varepsilon, \delta, M$ and $K$ such that

$$
E \exp \left\{\sum_{j=i+1}^{n} \varepsilon\left\|\varphi_{j}\right\|^{2+\delta}\right\} \leq M \exp \{K(n-i)\} \quad \forall n \geq i \geq 0
$$

L1'). The random process $F_{k} \triangleq \varphi_{k} \varphi_{k}^{\tau}$ has the following expansion:

$$
F_{k}=\sum_{j=0}^{\infty} A_{j} Z_{k-j}+D_{k}, \quad \sum_{j=0}^{\infty}\left\|A_{j}\right\|<\infty
$$

where $\left\{Z_{k}\right\}$ is an independent process such that $\left\{Z_{j}, j \geq\right.$ $k+1\}$ and $\left\{v_{j-1}, w_{j}, j \leq k\right\}$ are independent and satisfies

$$
\sup _{k} E \exp \left\{\alpha\left\|Z_{k}\right\|^{1+\delta}\right\}<\infty, \quad \text { for some } \quad \alpha>0, \delta>0
$$

and where $\left\{D_{k}\right\}$ is a bounded deterministic process.

Theorem 5.1. Let Conditions $\mathrm{P} 1$ and P3 of Section 3 be satisfied. If either L1) or L1') above holds, then Conditions (A1)-(A4) of Theorem 4.1 hold (for all $p \geq 1, q \geq 1$ ) and Theorem 3.1 is true for the LMS case.

Proof. First, in the LMS case, Conditions P1 and L1 (or L1') ensure that Condition (A1) of Theorem 4.1 holds for all $p \geq 1$ (cf. [9], Theorem 3.3). Next, when L1) holds, by Example 4.1 we know that Condition (A2) is true for all $q \geq$ 1. Also, when L1') holds, by the assumed independency we have for all $q \geq 1$,

$$
\begin{aligned}
& \left\|E\left[F_{k} \mid \mathcal{F}_{k-m}\right]-E F_{k}\right\|_{q}=\left\|\sum_{j=m}^{\infty}\left[A_{j} Z_{k-j}-E A_{j} Z_{k-j}\right]\right\|_{q} \\
& =O\left(\sum_{j=m}^{\infty}\left\|A_{j}\right\|\right), \quad \forall m \geq 1 .
\end{aligned}
$$

Hence (A2) holds again for all $q \geq 1$.

Moreover, Conditions (A3) and (A4) hold obviously in the present case. Finally, by 39, the result of Theorem 3.1(in the LMS case) follows directly from Theorem 4.1. This completes the proof.

\section{B. Analysis of $R L S$}

For the RLS algorithm defined by (11), (13) and (14), let us introduce the following two kinds of excitation conditions:

R1). There exist constants $h>0, c>0, \delta>0$ such that

$$
P\left\{\lambda_{\min }\left(\sum_{i=k+1}^{k+h} \varphi_{i} \varphi_{i}^{\tau}\right) \geq c \mid \mathcal{F}_{k}\right\}>\delta, \quad \forall k
$$

R1'). There exists $h>0$ such that

$$
\left.\sup _{k} E\left[\lambda_{\min }\left(\sum_{i=k+1}^{k+h} \varphi_{i} \varphi_{i}^{\tau}\right)\right]\right]^{-t}<\infty, \quad \forall t \geq 1 .
$$

The following weak dependence condition will also be used:

R2). There exists a number $t \geq 5$, such that $\left\|\varphi_{k}\right\|_{4 t}=$ $O(1)$, and that

$$
\left\|E\left[\varphi_{k} \varphi_{k}^{\tau} \mid \mathcal{F}_{k-m}\right]-E \varphi_{k} \varphi_{k}^{\tau}\right\|_{2 t} \leq \phi(m), \quad \forall k, m
$$

where $\phi(m) \rightarrow 0$ as $m \rightarrow \infty$.

Remark 5.1. Detailed discussions and investigations on the above first two conditions can be found in [10] and [17]. It has been shown in [10] that if Condition P1 and (21) in Section 3 hold, then R1) is true; Also, if $\left\{\varphi_{k}\right\}$ is generated by a linear state space model as in Example 4.2, then R1') can be verified (cf.[17]). Moreover, Condition R2) has been discussed in the last section.

Theorem 5.2. Let Conditions R1 ( or R1') and R2 above be satisfied. Then Conditions (A1)-(A3) of Theorem 4.1 hold (for any $p<2 t, q<t$ ) and Theorem 3.1 is true for the RLS case.

Proof. First, note that

$$
\prod_{j=i+1}^{k}\left(I-\mu F_{j}\right)=(1-\mu)^{k-i} P_{k+1} P_{i+1}^{-1}, \quad \forall k \geq i
$$

and

$$
P_{k}^{-1}=(1-\mu) P_{k-1}^{-1}+\mu \varphi_{k} \varphi_{k}^{\tau} .
$$

From this and condition R2 it follows that

$$
\left\|P_{k}^{-1}\right\|_{2 t}=O(1), \quad \forall \mu \in(0,1) .
$$

Also, by Theorem 1 in $[10]$, there is $\mu^{*} \in(0,1)$ such that

$$
\left\{P_{k}\right\} \in \mathcal{L}_{s}\left(\mu^{*}\right), \quad \forall s \geq 1
$$

Combining (40), (42), (43), we get

$$
\left\{F_{k}\right\} \in \mathcal{S}_{p}, \quad \forall p<2 t .
$$


Now, define $\left(\bar{P}_{0}=P_{0}\right)$

$$
\bar{P}_{k}^{-1}=(1-\mu) \bar{P}_{k-1}^{-1}+\mu E\left(\varphi_{k} \varphi_{k}^{\tau}\right) .
$$

Since either R1 or R1' implies P1 in Section 3 (cf.[10]), by a similar (actually simpler) argument as that used for the proof of (43) we know that $\left\|\bar{P}_{k}\right\|=O(1)$. We next prove that

$$
\left\|P_{k}^{-1}-\bar{P}_{k}^{-1}\right\|_{2 t}=O(\delta(\mu)), \quad \delta(\mu)=\min _{m \geq 1}\{\sqrt{\mu} m+\phi(m)\} .
$$

First, by (41) and (45)

$$
P_{k}^{-1}-\bar{P}_{k}^{-1}=\mu \sum_{i=1}^{k}(1-\mu)^{k-i}\left[\varphi_{i} \varphi_{i}^{\tau}-E \varphi_{i} \varphi_{i}^{\tau}\right]
$$

For any fixed $m \geq 1$, by denoting

$\delta_{j}(i)=E\left[\varphi_{i} \varphi_{i}^{\tau} \mid \mathcal{F}_{i-j}\right]-E\left[\varphi_{i} \varphi_{i}^{\tau} \mid \mathcal{F}_{i-j-1}\right] \quad, \quad 0 \leq j \leq m-1$

we have

$$
\begin{aligned}
& \varphi_{i} \varphi_{i}^{\tau}-E \varphi_{i} \varphi_{i}^{\tau} \\
& =\sum_{j=0}^{m-1} \delta_{j}(i)+\left\{E\left[\varphi_{i} \varphi_{i}^{\tau} \mid \mathcal{F}_{i-m}\right]-E\left[\varphi_{i} \varphi_{i}^{\tau}\right]\right\}
\end{aligned}
$$

Now, since for each $j$, the sequence $\left\{\delta_{j}(i), i \geq 1\right\}$ is a martingale difference, we can apply Lemma A.2 in the Appendix to each such $\left\{\delta_{j}(i), i \geq 1\right\}$ to obtain

$$
\mu\left\|\sum_{i=1}^{k}(1-\mu)^{k-i} \sum_{j=0}^{m-1} \delta_{j}(i)\right\|_{2 t}=O(\sqrt{\mu} m)
$$

Also, by our assumption

$$
\mu\left\|\sum_{i=1}^{k}(1-\mu)^{k-i}\left\{E\left[\varphi_{i} \varphi_{i}^{\tau} \mid \mathcal{F}_{i-m}\right]-E\left[\varphi_{i} \varphi_{i}^{\tau}\right]\right\}\right\|_{2 t} \leq \phi(m)
$$

Hence, (46) follows from (47)-(50) immediately.

Similar to the proof of (44), it is evident that

$$
\left\{\bar{P}_{k} \varphi_{k} \varphi_{k}^{\tau}\right\} \in \mathcal{S} .
$$

Now

$$
\left\|P_{k}-\bar{P}_{k}\right\| \leq\left\|P_{k}\right\| \cdot\left\|P_{k}^{-1}-\bar{P}_{k}^{-1}\right\| \cdot\left\|\bar{P}_{k}\right\|,
$$

from this, (43) and (46) it follows that

$$
\left\|P_{k}-\bar{P}_{k}\right\|_{s}=O(\delta(\mu)), \quad \forall s<2 t,(\text { for small } \mu),
$$

Hence, by Proposition 4.2 and (51), we know that $\left\{F_{k}\right\} \in$ $\mathcal{S}$. This in conjunction with (44) verifies Condition (A1).

Now, by (52) and R2 from Proposition 4.3 it is evident that Condition (A2) holds for any $q<t$.

To prove (A3), first note that for any $q<t$, (44) implies

$$
\left\{F_{k}\right\} \in \mathcal{L}_{2 q}\left(\mu^{*}\right), \text { for some } \mu^{*}>0 .
$$

So we need only to prove that

$$
\left\{L_{i}\right\} \in \mathcal{L}_{r}\left(\mu^{*}\right), \quad \text { for } \quad r>\left(\frac{1}{2}-\frac{1}{2 t}-\frac{3}{2 t}\right)^{-1}=\frac{2 t}{t-4} .
$$

This is true since by (43) and $\left\|\varphi_{k}\right\|_{4 t}=O(1)$,

$$
\left\{L_{i}\right\}=\left\{P_{i} \varphi_{i}\right\} \in \mathcal{L}_{r}\left(\mu^{*}\right), \quad \forall r<4 t,
$$

and since $4 t>\frac{2 t}{t-4}$. Hence (A3) holds.

Thus, by taking $t=\infty$ in the above argument, we see that Conditions (A1) and (A2) hold for all $p \geq 1$ and all $q \geq 1$. Hence Theorem 4.1 can be applied to prove Theorem 3.1 for the RLS case, while the expression for $\Pi_{k}$ will follow from Corollary 4.1 if we can prove that

$$
\left\|P_{k}-R_{k}\right\|_{s}=O(\delta(\mu)), \quad s=\frac{t}{t-1}
$$

where $P_{k}$ and $R_{k}$ are respectively defined by (14) and (24). Furthermore, by (52), it is clear that (53) will be true if

$$
\left\|R_{k}-\bar{P}_{k}\right\|=O(\delta(\mu))
$$

holds. However, this can be verified by using the definitions for $R_{k}$ and $\bar{P}_{k}$ (see Appendix B). Hence the proof is complete.

\section{Analysis of the KF algorithm}

Among the three basic algorithms described in Section 2 , the KF algorithm defined by (11), (16) and (17) is the most complicated one to analyze. Let us now introduce the following two conditions on stochastic excitation and weak dependence.

K1). There are constants $h>0$ and $\lambda \in(0,1)$ (independent of $\delta$ ) such that

$$
\left\{\frac{\lambda_{k}}{1+b_{k h+1}}\right\} \in \mathcal{S}^{0}(\lambda),
$$

where $\mathcal{S}^{0}(\lambda)$ is defined by (30), and $\lambda_{k}$ and $b_{k}$ are defined as follows: $\left(\mathcal{G}_{k}\right.$ is as before the sigma-algebra generated by $\left\{\varphi_{i}, i \leq k\right\}$.)

$$
\begin{aligned}
& \lambda_{k} \triangleq \lambda_{\min }\left\{E\left[\frac{1}{1+h} \sum_{i=k h+1}^{(k+1) h} \frac{\varphi_{i} \varphi_{i}^{\tau}}{1+\left\|\varphi_{i}\right\|^{2}} \mid \mathcal{G}_{k h}\right]\right\}, \\
& b_{k}=(1-\delta) b_{k-1}+\delta\left(\left\|\varphi_{k}\right\|^{2}+1\right) \quad, \quad \delta \in(0,1)
\end{aligned}
$$

K2). There exists a number $t \geq 7$ together with a function $\phi(m) \rightarrow 0($ as $m \rightarrow \infty)$ such that $\left\|\varphi_{k}\right\|_{4 t}=O(1)$, and that

$$
\left\|E\left[\varphi_{k} \varphi_{k}^{\tau} \mid \mathcal{F}_{k-m}\right]-E \varphi_{k} \varphi_{k}^{\tau}\right\|_{t} \leq \phi(m) \quad \forall k, m .
$$

Remark 5.2. If Conditions $\mathrm{P} 1$ and $\mathrm{P} 2$ of Section 3 are satisfied, then both K1) and K2) above hold (cf.[10]); When P2 is replaced by, for example, the situation discussed in Example 4.2, then again, both K1) and K2) can be verified (cf. [8]). 
Theorem 5.3. Let Conditions $\mathrm{K} 1$ and $\mathrm{K} 2$ above be satisfied. Then Conditions (A1)-(A3) of Theorem 4.1 hold (for any $p<2 t, q<\frac{4 t}{7}$ ) and Theorem 3.1 is true for the KF case.

The proof is prefaced by several lemmas. First, we need some results proved in the companion paper ([9], Theorem 3.5, Lemmas 7.1 and 7.2 ), which are collected into the following lemma for convenience of reference.

Lemma 5.1. For the KF algorithm defined by (11), (16) and (17), let Condition K1) be satisfied, and $\left\|\varphi_{k}\right\|_{4 t}=O(1)$ for some $t \geq \frac{1}{2}$. Then

$$
\begin{array}{ll}
\text { (i) } & \left\{P_{k}\right\} \in \mathcal{L}_{s}\left(\mu^{*}\right), \quad \forall \mu^{*} \in(0,1), \forall s \geq 1 \\
\text { (ii) } & \left\{P_{k}^{-1}\right\} \in \mathcal{L}_{2 t}\left(\mu^{*}\right), \quad \forall \mu^{*} \in(0,1) \\
\text { (iii) } & \left\{F_{k}\right\} \in \mathcal{S}_{p}, \quad \forall p<2 t .
\end{array}
$$

In order to apply Proposition 4.3, our main objective is then to show that $\left\{P_{k}\right\}$ can be "approximated" by a deterministic process $\left\{\bar{P}_{k}\right\}$ defined by

$$
\begin{aligned}
& \bar{P}_{k}=\left[\bar{P}_{k-1}^{-1}+\mu R^{-1} E\left(\varphi_{k} \varphi_{k}^{\tau}\right)\right]^{-1}+\mu Q, \\
& \bar{P}_{0}=P_{0} .
\end{aligned}
$$

First, by a similar (actually simplified) argument as that for Lemma 5.1, the following lemma can be established (details are not repeated).

Lemma 5.2. Assume that $\left\|\varphi_{k}\right\|_{2}=O(1)$, and that there is an integer $h>0$ such that

$$
\inf _{k} \lambda_{\min }\left\{\sum_{i=k+1}^{k+h} E\left(\varphi_{i} \varphi_{i}^{\tau}\right)\right\}>0 .
$$

Then the following three properties hold:
(i). $\left\|\bar{P}_{k}\right\|=O(1)$

$$
\forall \mu \in(0,1) \text {. }
$$
(ii). $\left\|\bar{P}_{k}^{-1}\right\|=O(1)$,
$\forall \mu \in(0,1)$.
(iii). $\left\{\left(\bar{P}_{k}-\mu Q\right) R^{-1} \varphi_{k} \varphi_{k}^{\tau}\right\} \in \mathcal{S}\left(\mu^{*}\right), \quad \forall \mu^{*} \in(0,1)$.

The last assertion (iii) above corresponds to that in Lemma 5.1 , because by (16)-(17), $F_{k} \triangleq L_{k} \varphi_{k}^{\tau}$ can be rewritten as

$$
F_{k}=\left(P_{k}-\mu Q\right) R^{-1} \varphi_{k} \varphi_{k}^{\tau}
$$

To be able to estimate the "distance" between $P_{k}$ and $\bar{P}_{k}$, we need some auxiliary results.

Define $\forall k \geq i$

$$
\begin{aligned}
& \Phi(k+1, i)=\left(I-\mu F_{k}\right) \Phi(k, i), \Phi(i, i)=I, \\
& \Psi(k+1, i)=\left(I-\mu \bar{F}_{k}\right) \Psi(k, i), \Psi(i, i)=I,
\end{aligned}
$$

where $\bar{F}_{k} \triangleq\left(\bar{P}_{k}-\mu Q\right) R^{-1} E\left(\varphi_{k} \varphi_{k}^{\tau}\right)$.

Lemma 5.3. Let $\left\{e_{k}\right\} \in \mathcal{M}_{r}, r \geq 1$,

$\left\{\bar{F}_{i}\right\} \in \mathcal{S}\left(\mu^{*}\right), \quad \sup _{i \geq 0}\|\Phi(k, i)\|_{q}=O(1), \forall \mu \in\left(0, \mu^{*}\right]$,
Then for $s \triangleq\left(r^{-1}+q^{-1}+t^{-1}\right)^{-1}$,

$\left\|\sum_{i=0}^{k} \Phi(k+1, i+1) e_{i} \Psi^{\tau}(k+1, i+1)\right\|_{s}=O\left(\mu^{-\frac{1}{2}}\right), \forall \mu \in\left(0, \mu^{*}\right]$.

The proof is essentially the same as that for Lemma A.2 in the Appendix, and hence details are not repeated.

Lemma 5.4. Let Condition K1) hold, and $\left\|\varphi_{k}\right\|_{4 t}=$ $O(1), t \geq 1$. If $\left\{e_{k}\right\} \in \mathcal{M}_{r}$, then for $s<\left(r^{-1}+\frac{3}{4 t}\right)^{-1}$,

$\left\|\sum_{i=0}^{k} \Phi(k+1, i+1) e_{i} \Psi^{\tau}(k+1, i+1)\right\|_{s}=O\left(\mu^{-\frac{1}{2}}\right)$, for small $\mu$.

Proof. We need only to verify the conditions of Lemma 5.3. First of all, K1) implies $\left\{\lambda_{k}\right\} \in \mathcal{S}^{0}(\lambda), \lambda \in(0,1)$. So by Theorem 2.2 in [8]

$$
\inf _{k} \lambda_{\min }\left\{\sum_{i=k h_{0}+1}^{(k+1) h_{0}} E \lambda_{i}\right\}>0, \quad \text { for some } h_{0}>0 .
$$

which implies that there exists $h_{1}>0$, such that

$$
\inf _{k} \lambda_{\min }\left\{\sum_{i=k h_{1}+1}^{(k+1) h_{1}} E\left[\frac{\varphi_{i} \varphi_{i}^{\tau}}{1+\left\|\varphi_{i}\right\|^{2}}\right]\right\}>0 .
$$

hence, we have

$$
\inf _{k} \lambda_{\min }\left\{\sum_{i=k h_{1}+1}^{(k+1) h_{1}} E\left[\varphi_{i} \varphi_{i}^{\tau}\right]\right\}>0
$$

Therefore, (55) is true and Lemma 5.2 is applicable.

Now, by Theorem 2.4 in [8],

$$
\|\Phi(k, i)\| \leq\left\{\left\|P_{k}\right\| \cdot\left\|P_{i}^{-1}\right\|\right\}^{\frac{1}{2}}
$$

So, by Lemma 5.1,

$$
\sup _{k \geq i \geq 0}\|\Phi(k, i)\|_{q}=O(1), \quad \forall q<4 t .
$$

Moreover, by (56) and Lemma 5.1(i)

$$
\left\{F_{i}\right\} \in \mathcal{L}_{p}\left(\mu^{*}\right), \quad \forall p<2 t .
$$

Now, by (59), (60) and Lemma 5.2(iii), it follows from Lemma 5.3 that the desired result is true.

$$
\#
$$

Lemma 5.5 Let $\left\{P_{k}\right\}$ and $\left\{\bar{P}_{k}\right\}$ be respectively defined by (17) and (54). Then

$$
\begin{aligned}
P_{k}-\bar{P}_{k}= & \left(I-\mu F_{k}\right)\left(P_{k-1}-\bar{P}_{k-1}\right)\left(I-\mu \bar{F}_{k}\right)^{\tau} \\
& +\mu R^{-1}\left(P_{k}-\mu Q\right)\left[E\left(\varphi_{k} \varphi_{k}^{\tau}\right)-\varphi_{k} \varphi_{k}^{\tau}\right]\left(\bar{P}_{k}-\mu Q\right)
\end{aligned}
$$


where $F_{k}$ is defined by (56) and $\bar{F}_{k}$ is defined as in (58).

Proof. Denote

$$
\begin{aligned}
& Q_{k} \triangleq\left[P_{k-1}^{-1}+\mu R^{-1} \varphi_{k} \varphi_{k}^{\tau}\right] \\
& \bar{Q}_{k} \triangleq\left[\bar{P}_{k-1}^{-1}+\mu R^{-1} E\left(\varphi_{k} \varphi_{k}^{\tau}\right)\right] .
\end{aligned}
$$

Then by (17) and (54),

$$
\begin{aligned}
& P_{k}-\bar{P}_{k}=Q_{k}^{-1}-\bar{Q}_{k}^{-1}=Q_{k}^{-1}\left[\bar{Q}_{k}-Q_{k}\right] \bar{Q}_{k}^{-1} \\
& =Q_{k}^{-1}\left\{\bar{P}_{k-1}^{-1}-P_{k-1}^{-1}+\mu R^{-1}\left[E\left(\varphi_{k} \varphi_{k}^{\tau}\right)-\varphi_{k} \varphi_{k}^{\tau}\right]\right\} \bar{Q}_{k}^{-1} \\
& =Q_{k}^{-1} P_{k-1}^{-1}\left(P_{k-1}-\bar{P}_{k-1}\right) \bar{P}_{k-1}^{-1} \bar{Q}_{k}^{-1} \\
& \quad+\mu R^{-1} Q_{k}^{-1}\left[E\left(\varphi_{k} \varphi_{k}^{\tau}\right)-\varphi_{k} \varphi_{k}^{\tau}\right] \bar{Q}_{k}^{-1}
\end{aligned}
$$

But, it is not difficult to verify that (via the matrix inverse formula),

$$
Q_{k}^{-1} P_{k-1}^{-1}=I-\mu F_{k}
$$

and

$$
\bar{P}_{k-1}^{-1} \bar{Q}_{k}^{-1}=I-\mu \bar{F}_{k}^{\tau}
$$

Hence, the desired result follows from (61) directly.

Lemma 5.6. Let Conditions K1) and K2) be satisfied. Then

$$
\left\|P_{k}-\bar{P}_{k}\right\|_{s}=O(\delta(\mu)), \quad \forall s<\frac{4 t}{5}
$$

holds for all small $\mu$, where $\delta(\mu)=\min _{m \geq 1}\{\sqrt{\mu} m+\phi(m)\}$.

Proof. Set

$$
\begin{aligned}
& H_{k}=R^{-1}\left(P_{k}-\mu Q\right) \\
& X_{k}=\left(\varphi_{k} \varphi_{k}^{\tau}\right)\left(\bar{P}_{k}-\mu Q\right)
\end{aligned}
$$

Then by (57), (58) and Lemma 5.5 we have $(\forall k \geq 1, \forall m \geq$ 1),

$$
\begin{aligned}
& P_{k}-\bar{P}_{k} \\
& =\mu \sum_{i=1}^{k} \Phi(k+1, i+1) H_{i}\left[E X_{i}-X_{i}\right] \Psi^{\tau}(k+1, i+1) \\
& =\mu \sum_{i=1}^{k} \Phi(k+1, i+1)\left[H_{i-m}\right. \\
& \left.\quad+\left(H_{i}-H_{i-m}\right)\right]\left[E X_{i}-X_{i}\right] \Psi^{\tau}(k+1, i+1) .
\end{aligned}
$$

Note that by (17), $\forall i \geq 1, \forall m \geq 1$,

$$
\begin{aligned}
H_{i} & -H_{i-m}=R^{-1}\left(P_{i}-P_{i-m}\right) \\
& =\mu R^{-1} \sum_{k=i-m+1}^{i}\left[-\frac{P_{k-1} \varphi_{k} \varphi_{k}^{\tau} P_{k-1}}{R+\mu \varphi_{k}^{\tau} P_{k-1} \varphi_{k}}+Q\right] .
\end{aligned}
$$

From this and Lemma 5.1(i), we have $\forall m \geq 1$

$$
\left\|H_{i}-H_{i-m}\right\|_{p}=O(\mu m), \quad \forall p<2 t, \mu \in(0,1),
$$

Hence, by Lemma 5.2(iii), (59) and the Hölder inequality, for any $s<\frac{4 t}{5}$

$$
\begin{aligned}
& \| \sum_{i=1}^{k} \Phi(k+1, i+1)\left(H_{i}-H_{i-m}\right) \\
\times & {\left[E X_{i}-X_{i}\right] \Psi^{\tau}(k+1, i+1) \|_{s} } \\
= & O\left(\mu m \sum_{i=1}^{k}(1-\mu \alpha)^{(k-i)}\right), \quad(\text { for some } \quad \alpha \in(0,1)) \\
= & O(m), \quad \forall m \geq 1 .
\end{aligned}
$$

Now, consider the following decomposition :

$$
\begin{aligned}
X_{i}-E X_{i} & =\sum_{j=0}^{m-1} \delta_{j}(i)+\left\{E\left[X_{i} \mid \mathcal{G}_{i-m}\right]-E X_{i}\right\} \\
\delta_{j}(i) & \triangleq E\left[X_{i} \mid \mathcal{G}_{i-j}\right]-E\left[X_{i} \mid \mathcal{G}_{i-j-1}\right], \quad 0 \leq j \leq m-1 \\
\mathcal{G}_{i} & =\sigma\left\{\varphi_{s}, \quad s \leq i\right\}
\end{aligned}
$$

and note that $\left\{H_{i-m} \delta_{j}(i), \mathcal{G}_{i-j}, i \geq 1\right\}$ is a martingale difference for each $0 \leq j \leq m-1$, and that

$$
\sup _{i, j}\left\|H_{i-m} \delta_{j}(i)\right\|_{p}=O(1), \quad \forall p<2 t, 1 \leq j \leq m-1
$$

then by Lemma 5.4 we have for small $\mu>0$ and for $s<\frac{4 t}{5}$,

$$
\begin{aligned}
& \sum_{j=0}^{m-1}\left\|\sum_{i=1}^{k} \Phi(k+1, i+1)\left[H_{i-m} \delta_{j}(i)\right] \Psi^{\tau}(k+1, i+1)\right\|_{s} \\
= & O\left(\mu^{-\frac{1}{2}} m\right)
\end{aligned}
$$

Next, by Condition K2), (59) and Lemma 5.2(iii), it follows that for any $s<\frac{4 t}{5}$,

$$
\begin{aligned}
& \| \sum_{i=1}^{k} \Phi(k+1, i+1) H_{i-m}\left\{E\left[X_{i} \mid \mathcal{G}_{i-m}\right]-E X_{i}\right\} \\
& \quad \times \Psi^{\tau}(k+1, i+1) \|_{s}=O\left(\mu^{-1} \phi(m)\right)
\end{aligned}
$$

Finally, substituting (65)- (68) into (62) we see that for small $\mu$ and any $m \geq 1$

$$
\left\|P_{k}-\bar{P}_{k}\right\|_{s}=O(\sqrt{\mu} m+\phi(m)), \quad \forall s<\frac{4 t}{5} .
$$

Hence Lemma 5.6 is true.

Proof of Theorem 5.3. First, we prove Condition (A1). By Lemma 5.6, Lemma 5.2(iii) and (56) from Proposition 4.2 we know that $\left\{F_{k}\right\} \in \mathcal{S}$. Consequently, by Lemma 5.1, we have

$$
\left\{F_{k}\right\} \in \mathcal{S}_{p} \bigcap \mathcal{S}, \quad \forall p<2 t
$$

which verifies condition (A1).

Next, by Proposition 4.3 and Lemma 5.6, it can be seen that (A2) holds for any $q<\frac{4 t}{7}$. 
Finally, to verify Condition (A3), we note that by Lemma 5.1(i),

$$
\left\{L_{i}\right\} \in \mathcal{L}_{r}\left(\mu^{*}\right), \quad \forall r<4 t, \quad \mu^{*} \in(0,1)
$$

and

$$
\left\{F_{i}\right\} \in \mathcal{L}_{2 q}\left(\mu^{*}\right), \quad \forall q<\frac{4 t}{7}, \quad \mu^{*} \in(0,1) .
$$

But $\left(\frac{1}{2}-\frac{1}{2 t}-\frac{3}{2} \cdot \frac{2}{t}\right)^{-1}<4 t$, hence condition (A3) is also true.

Thus, Theorem 4.1 can be applied to prove Theorem 3.1 (by taking $t=\infty$ ), while the expresion for $\Pi_{t+1}$ will follow from Corollary 4.1 if we can show that

$$
\left\|P_{k} R^{-1}-R_{k}\right\|_{s}=O(\delta(\mu)), \forall s<\frac{4 t}{5} .
$$

However, this follows directly from Lemma 5.6 since it can be verified (See Appendix B) that

$$
\left\|R_{k}-\bar{P}_{k} R^{-1}\right\|=O(\mu) .
$$

This completes the proof of Theorem 5.3.

\section{Conclusions}

We have in this article presented a number of results, by which the true covariance matrix of the parameter tracking error can be approximated by a matrix that can be computed by a much simpler equation. As mentioned above, there is a considerable literature on this problem. We may point to the following aspects of how the results of this paper extends and contains earlier ones.

- The approximation in Therems 3.1 and 4.1 is explicit. It involves the true error covariance matrix and the approximating one. The result is not asymptotic. (However it is of interest only for small gains $\mu$.) It is applicable both during the transient and over infinite time horisons.

- We have treated the whole family of the most commonly used tracking algorithms in one general result (Theorem 3.1).

- This includes what appears to be the first formal treatment of the Kalman Filter as a tracking algorithm in this respect.

- For the LMS case, the regressors are not assumed to be bounded (Theorem 5.1).

- For the RLS case, the Riccati equation for calculating $\Pi_{k}$ is simpler than those earlier used (See (14).)

- We have also, in Theorem 4.1 given a general result on the tracking error under quite weak assumptions. Together with the results of Section V, this may serve as a tool kit for building specialized results for particular algorithms.

The basic result is quite easy to understand, and its practical implications for dealing with the key features of tracking algorithms are quite important. The basis for the important compromise between tracking ability and noise sensitivity lies in these expressions. Nevertheless, the analysis and the proof of the result turn out to be surprisingly technically complicated.
Appendix A: Proof of Theorem 4.1

We first prove several lemmas.

Lemma A.1 Let $\alpha \in(0,1)$ be a constant. Then $\forall \mu \in$ $(0,1)$$$
\sup _{k \geq 0}(1-\alpha \mu)^{k} \sqrt{k}=O\left(\mu^{-\frac{1}{2}}\right)
$$$$
\sum_{k=0}^{\infty}(1-\alpha \mu)^{k} k=O\left(\mu^{-2}\right)
$$$$
\sum_{k=0}^{\infty}(1-\alpha \mu)^{k} \sqrt{k}=O\left(\mu^{-\frac{3}{2}}\right)
$$

where the " $O$ " constant depends only on $\alpha$.

Proof. Denote $\beta=e^{-\alpha}<1$, then we have

$$
(1-\alpha \mu)^{k} \leq \beta^{k \mu}, \quad \forall \mu \in(0,1) .
$$

Now, note that $\sup _{x \geq 0} \beta^{x} \sqrt{x}<\infty$, then replace $x$ by $k \mu$ gives assertion (i). To prove (ii), observe that

$$
\begin{aligned}
\sum_{k=0}^{\infty} \beta^{k \mu} k & =\frac{\partial}{\partial \mu}\left(\sum_{k=0}^{\infty} \frac{1}{\log \beta} \beta^{k \mu}\right) \\
& =\frac{1}{\log \beta} \frac{\partial}{\partial \mu}\left(\frac{1}{1-\beta^{\mu}}\right) \leq \frac{1}{(\beta \log \beta)^{2}} \frac{1}{\mu^{2}}
\end{aligned}
$$

Hence (ii) is also true. Finally, (iii) follows from (ii) via Schwarz inequality.

Now, define

$\Phi(k+1, i)=\left(I-\mu F_{k}\right) \Phi(k, i), \quad \Phi(i, i)=I, \quad \forall k \geq i \geq 0$.

Lemma A.2 Let $\left\{e_{k}\right\} \in \mathcal{M}_{r}, r \geq 1$, and $\left\{F_{k}\right\} \in$ $\mathcal{S}_{p}\left(\mu^{*}\right) \cap \mathcal{L}_{t}\left(\mu^{*}\right), p \geq 1, t \geq 1, \mu^{*} \in(0,1)$. Then for $s \triangleq\left(r^{-1}+p^{-1}+t^{-1}\right)^{-1}$,

$$
\left\|\sum_{i=0}^{k} \Phi(k+1, i+1) e_{i}\right\|_{s}=O\left(\mu^{-\frac{1}{2}}\right), \quad \forall \mu \in\left(0, \mu^{*}\right] .
$$

Proof. Set $S(k, i)=\sum_{j=i}^{k} e_{j}$, then by summation by parts,

$$
\begin{aligned}
\sum_{i=0}^{k} \Phi(k+1, i+1) e_{i} \\
=\sum_{i=0}^{k} \Phi(k+1, i+1)[S(k, i)-S(k, i+1)] \\
=\Phi(k+1,1) S(k, 0) \\
\quad+\sum_{i=1}^{k}[\Phi(k+1, i+1)-\Phi(k+1, i)] S(k, i) \\
=\Phi(k+1,1) S(k, 0)+\mu \sum_{i=1}^{k} \Phi(k+1, i+1) F_{i} S(k, i)
\end{aligned}
$$


So by the Hölder inequality and Lemma A.1,

$$
\begin{aligned}
& \left\|\sum_{i=0}^{k} \Phi(k+1, i+1) e_{i}\right\|_{s} \\
& \leq\|\Phi(k+1,1)\|_{p} \cdot\|S(k, 0)\|_{r} \\
& \quad+\mu \sum_{i=1}^{k}\|\Phi(k+1, i+1)\|_{p} \cdot\left\|F_{i}\right\|_{t} \cdot\|S(k, i)\|_{r}, \quad \text { A } \\
& =O\left([1-\alpha \mu]^{k} \sqrt{k}\right)+O\left(\mu \sum_{i=0}^{k}(1-\alpha \mu)^{k-i} \sqrt{k-i}\right) \\
& =O\left(\mu^{-\frac{1}{2}}\right)+O\left(\mu^{-\frac{1}{2}}\right)=O\left(\mu^{-\frac{1}{2}}\right), \quad \forall \mu \in\left(0, \mu^{*}\right] .
\end{aligned}
$$

Note that by (19),

$\widetilde{\theta}_{k+1}=\Phi(k+1,0) \widetilde{\theta}_{0}+\mu \sum_{i=0}^{k} \Phi(k+1, i+1)\left[-\mu L_{i} v_{i}+\gamma w_{i+1}\right]$.

Applying Lemma A.2 we immediately have

Lemma A.3 Let $\left\{L_{i} v_{i}\right\} \in \mathcal{M}_{r},\left\{w_{i}\right\} \in \mathcal{M}_{r},\left\{F_{k}\right\} \in$ $\mathcal{S}_{p}\left(\mu^{*}\right) \cap \mathcal{L}_{t}\left(\mu^{*}\right), r \geq 1, p \geq 1, t \geq 1, \mu^{*} \in(0,1)$. Then for $s \triangleq\left(r^{-1}+p^{-1}+t^{-1}\right)^{-1}$, and $\forall \mu \in\left(0, \mu^{*}\right]$,

$$
\left\|\widetilde{\theta}_{k+1}\right\|_{s}=O\left(\sqrt{\mu}+\frac{\gamma}{\sqrt{\mu}}+(1-\alpha \mu)^{k}\right)
$$

holds for all $k \geq 1$, where $\alpha \in(0,1)$ is a constant.

Now, let us introduce a new sequence $\left\{\bar{\theta}_{k}\right\}$ :

$$
\begin{aligned}
\bar{\theta}_{k+1} & =\left(I-\mu E\left[F_{k}\right]\right) \bar{\theta}_{k}-\mu L_{k} v_{k}+\gamma w_{k+1}, \\
\bar{\theta}_{0} & =\widetilde{\theta}_{0}
\end{aligned}
$$

Similar to the proof above, we have

Lemma A.4 Let $\left\{L_{i} v_{i}\right\} \in \mathcal{M}_{r},\left\{w_{i}\right\} \in \mathcal{M}_{r}, r \geq 1$, $\left\{F_{i}\right\} \in \mathcal{S}\left(\mu^{*}\right), \mu^{*} \in(0,1)$. Then

$$
\left\|\bar{\theta}_{k+1}\right\|_{r}=O\left(\sqrt{\mu}+\frac{\gamma}{\sqrt{\mu}}+(1-\alpha \mu)^{k}\right), \quad \forall \mu \in\left(0, \mu^{*}\right] .
$$

where $\alpha \in(0,1)$ is a constant (without loss of generality, it may be taken as the same as that in Lemma A.3).

Proof of Theorem 4.1. By (A.2) and Condition (A4) it is evident that

$$
\hat{\Pi}_{k+1}=E\left[\bar{\theta}_{k+1} \bar{\theta}_{k+1}^{\tau}\right], \quad \forall k \geq 0 .
$$

Hence by Schwarz inequality,

$$
\begin{aligned}
& \left\|E\left[\widetilde{\theta}_{k+1} \widetilde{\theta}_{k+1}^{\tau}\right]-\hat{\Pi}_{k+1}\right\| \\
& =\left\|E\left[\widetilde{\theta}_{k+1} \widetilde{\theta}_{k+1}^{\tau}-\bar{\theta}_{k+1} \bar{\theta}_{k+1}^{\tau}\right]\right\| \\
& =\left\|E\left[\left(\widetilde{\theta}_{k+1}-\bar{\theta}_{k+1}\right) \widetilde{\theta}_{k+1}^{\tau}+\bar{\theta}_{k+1}\left(\widetilde{\theta}_{k+1}^{\tau}-\bar{\theta}_{k+1}^{\tau}\right)\right]\right\| \\
& \leq\left\|\widetilde{\theta}_{k+1}-\bar{\theta}_{k+1}\right\|_{2} \cdot\left[\left\|\widetilde{\theta}_{k+1}\right\|_{2}+\left\|\bar{\theta}_{k+1}\right\|_{2}\right] .
\end{aligned}
$$

$$
\varepsilon_{k}(\alpha)=\sqrt{\mu}+\frac{\gamma}{\sqrt{\mu}}+(1-\alpha \mu)^{k} .
$$

By Lemmas A.3 and A.4, we know that

$$
\left\|\widetilde{\theta}_{k+1}\right\|_{2}+\left\|\bar{\theta}_{k+1}\right\|_{2}=O\left(\varepsilon_{k}(\alpha)\right) .
$$

So we need only to consider the first term on the RHS of (A.3).

By (19) and (A.2), it is seen that

$$
\widetilde{\theta}_{k+1}-\bar{\theta}_{k+1}=\left(I-\mu E\left[F_{k}\right]\right)\left(\widetilde{\theta}_{k}-\bar{\theta}_{k}\right)+\mu\left(E\left[F_{k}\right]-F_{k}\right) \widetilde{\theta}_{k},
$$

Define

$$
\Psi(k+1, i)=\left(I-\mu E\left[F_{k}\right]\right) \Psi(k, i), \quad \Psi(i, i)=I, \forall k \geq i .
$$

Then we have $(\forall k \geq 0)$,

$$
\begin{aligned}
\widetilde{\theta}_{k+1}-\bar{\theta}_{k+1}= & \mu \sum_{i=0}^{k} \Psi(k+1, i+1)\left(E\left[F_{i}\right]-F_{i}\right) \widetilde{\theta}_{i} \\
= & \mu \sum_{i=0}^{m-1} \Psi(k+1, i+1)\left(E\left[F_{i}\right]-F_{i}\right) \widetilde{\theta}_{i} \\
& +\mu \sum_{i=m}^{k} \Psi(k+1, i+1)\left(E\left[F_{i}\right]-F_{i}\right) \\
& \times\left\{\widetilde{\theta}_{i-m}+\left(\widetilde{\theta}_{i}-\widetilde{\theta}_{i-m}\right)\right\}
\end{aligned}
$$

where $m=m(\mu)$ which is defined by

$$
m(\mu)=\operatorname{argmin}_{m \geq 1}[\sqrt{\mu} m+\phi(m, \mu)] .
$$

Note that

$$
\sqrt{\mu} m(\mu) \leq \sqrt{\mu} m(\mu)+\phi(m(\mu), \mu) \leq \sqrt{\mu}+\phi(1, \mu)
$$

which implies that

$$
m(\mu) \leq 1+\frac{\phi(1, \mu)}{\sqrt{\mu}} \leq \frac{c}{\sqrt{\mu}}, \quad \forall \mu \in(0,1)
$$

for some constant $c>0$. Consequently, for any $\alpha \in(0,1)$,

$$
(1-\alpha \mu)^{-m(\mu)} \leq(1-\alpha \mu)^{\frac{-c}{\sqrt{\mu}}} \rightarrow 1, \quad \text { as } \quad \mu \rightarrow 0,
$$

hence $(1-\alpha \mu)^{-m(\mu)}, \mu \in(0,1)$, is a bounded function for any $\alpha \in(0,1)$. In the sequel, we will frequently use this fact without explanations, and will also drop the variable $\mu$ in $m(\mu)$ in what follows.

Now, denote

$$
s=\left(\frac{1}{r}+\frac{1}{p}+\frac{1}{2 q}\right)^{-1}
$$

Then by (A.4), Lemma A.3 and conditions (A1)-(A4) we have $\forall \mu \in\left(0, \mu^{*}\right]$,

$$
\left.\left\|\widetilde{\theta}_{k+1}\right\|_{s}=O\left(\varepsilon_{k}(\alpha)\right)\right),
$$


Note that the number $s$ defined by (A.8) satisfies $\left[s^{-1}+\right.$ $\left.(2 q)^{-1}\right]^{-1}>2$, we have by $($ A.9),

$$
\mu\left\|\sum_{i=0}^{m-1} \Psi(k+1, i+1)\left(E\left[F_{i}\right]-F_{i}\right) \widetilde{\theta}_{i}\right\|_{2}=O\left(\mu m \varepsilon_{k}(\alpha)\right)
$$

So, we need only to consider the last term in (A.6) for $k \geq m$.

Note that by (19), $\forall i \geq m$,

$$
\widetilde{\theta}_{i}-\widetilde{\theta}_{i-m}=\sum_{j=i-m}^{i-1}\left[-\mu F_{j} \widetilde{\theta}_{j}-\mu L_{j} v_{j}+\gamma w_{j+1}\right]
$$

So by denoting

$$
u=\left(\frac{1}{s}+\frac{1}{2 q}\right)^{-1}
$$

and applying the Hölder inequality to (A.11) and by noting that $\left\{-\mu L_{j} v_{j}+\gamma w_{j+1}\right\} \in \mathcal{M}_{r} \subset \mathcal{M}_{u}$, we have for any $i \geq m$,

$$
\begin{aligned}
& \left\|\widetilde{\theta}_{i}-\widetilde{\theta}_{i-m}\right\|_{u} \\
\leq & \sum_{j=i-m}^{i-1} \mu\left\|F_{j}\right\|_{2 q} \cdot\left\|\widetilde{\theta}_{j}\right\|_{s}+\left\|\sum_{j=i-m}^{i-1}\left(-\mu L_{j} v_{j}+\gamma w_{j+1}\right)\right\|_{u} \\
= & O\left(\sum_{j=i-m}^{i-1} \mu \varepsilon_{j}(\alpha)\right)+O(\sqrt{m}[\mu+\gamma]) \\
= & O\left(\sqrt{\mu} m\left[\sqrt{\mu}+\frac{\gamma}{\sqrt{\mu}}\right]\right)+O\left(\mu m(1-\alpha \mu)^{i-m}\right) .
\end{aligned}
$$

for $\mu \in\left(0, \mu^{*}\right]$.

By (A.8) and (A.12) and the definition of $r$ in Condition (A3), it is readily verified that $\frac{1}{2}=\frac{1}{2 q}+\frac{1}{u}$. Hence, by (A.13) and Condition (A1) we obtain $(\forall k \geq m)$,

$$
\begin{aligned}
& \mu\left\|\sum_{i=m}^{k} \Psi(k+1, i+1)\left(E\left[F_{i}\right]-F_{i}\right)\left(\widetilde{\theta}_{i}-\widetilde{\theta}_{i-m}\right)\right\|_{2} \\
& \leq \mu \sum_{i=m}^{k}\|\Psi(k+1, i+1)\| \cdot\left\|E\left[F_{i}\right]-F_{i}\right\|_{2 q} \cdot\left\|\widetilde{\theta}_{i}-\widetilde{\theta}_{i-m}\right\|_{u} \\
& =O\left(\sqrt{\mu} m\left[\sqrt{\mu}+\frac{\gamma}{\sqrt{\mu}}\right]\right)+O\left(\mu^{2} m(k-m)(1-\alpha \mu)^{k-m}\right) \\
& =O\left(\sqrt{\mu} m\left[\sqrt{\mu}+\frac{\gamma}{\sqrt{\mu}}\right]\right)+O\left(\mu^{2} m \sup _{k \geq 0}\left\{k(1-\alpha \mu)^{k}\right\}\right) \\
& =O\left(\sqrt{\mu} m\left[\sqrt{\mu}+\frac{\gamma}{\sqrt{\mu}}\right]\right)
\end{aligned}
$$

where for the last relationship we have used Lemma A.1(i).

Now, set for $j \geq 0, i \geq m$,

$$
\delta_{j}(i) \triangleq E\left[F_{i} \mid \mathcal{F}_{i-j}\right]-E\left[F_{i} \mid \mathcal{F}_{i-j-1}\right] .
$$

Then $\forall i \geq m$

$$
F_{i}-E F_{i}=\sum_{j=0}^{m-1} \delta_{j}(i)+E\left[F_{i} \mid \mathcal{F}_{i-m}\right]-E F_{i}
$$

For any fixed $0 \leq j \leq m-1$, denote $e_{i}=\delta_{j}(i) \widetilde{\theta}_{i-m}$, then it is obvious that $\left\{e_{i}, \mathcal{F}_{i-j}, i \geq m\right\}$ is a martingale difference sequence and that by (A.9) and the fact that $\frac{1}{2} \geq \frac{1}{2 q}+\frac{1}{s}$,

$$
\left\|e_{i}\right\|_{2} \leq 2\left\|F_{i}\right\|_{2 q} \cdot\left\|\widetilde{\theta}_{i-m}\right\|_{s}=O\left(\varepsilon_{i-m}(\alpha)\right), \quad i \geq m .
$$

Consequently, denote $S(k, i) \triangleq \sum_{j=i}^{k} e_{j}$, we have for any $k \geq$ $i>m$,

$$
\begin{aligned}
& \|S(k, i)\|_{2}=\left\{\sum_{j=i}^{k} E e_{j}^{2}\right\}^{\frac{1}{2}} \\
& =O\left(\sqrt{k-i+1}\left(\sqrt{\mu}+\frac{\gamma}{\sqrt{\mu}}\right)+(1-\alpha \mu)^{i-m} \mu^{\frac{-1}{2}}\right)
\end{aligned}
$$

Hence similar to (A.1) we have by (A.4) and Lemma A.1

$$
\begin{aligned}
\mu \| & \sum_{i=m}^{k} \Psi(k+1, i+1) \sum_{j=0}^{m-1} \delta_{j}(i) \widetilde{\theta}_{i-m} \|_{2} \\
\leq & \mu \sum_{j=0}^{m-1}\left\|\sum_{i=m}^{k} \Psi(k+1, i+1) e_{i}\right\|_{2} \\
\leq & \mu \sum_{j=0}^{m-1}\|\Psi(k+1, m+1)\| \cdot\|S(k, m)\|_{2} \\
& +\mu^{2} \sum_{j=0}^{m-1} \sum_{i=m+1}^{k}\|\Psi(k+1, i+1)\| \cdot\left\|E F_{i}\right\| \cdot\|S(k, i)\|_{2} \\
= & O\left(\mu m ( \sqrt { \mu } + \frac { \gamma } { \sqrt { \mu } } ) \left\{(1-\alpha \mu)^{k-m} \sqrt{k-m}\right.\right. \\
& \left.\left.+\mu \sum_{i=m+1}^{k}(1-\alpha \mu)^{k-i} \sqrt{k-i}\right\}\right) \\
& +O\left(\sqrt{\mu} m\left(1-\frac{\alpha}{2} \mu\right)^{k-m}\right) \\
= & O\left(\sqrt{\mu} m \varepsilon_{k}\left(\frac{\alpha}{2}\right)\right), \quad \forall k \geq m .
\end{aligned}
$$

Note that by (A.8) and the definition of $r$ in Condition (A3), we have $\frac{1}{2}=\frac{1}{q}+\frac{1}{s}$. Hence by (A.9) and Condition A2,

$$
\begin{aligned}
& \mu\left\|\sum_{i=m}^{k} \Psi(k+1, i+1)\left\{E\left[F_{i} \mid \mathcal{F}_{i-m}\right]-E F_{i}\right\} \widetilde{\theta}_{i-m}\right\|_{2} \\
& \leq \mu \sum_{i=m}^{k}\|\Psi(k+1, i+1)\| \cdot\left\|E\left[F_{i} \mid \mathcal{F}_{i-m}\right]-E F_{i}\right\|_{q} \cdot\left\|\widetilde{\theta}_{i-m}\right\|_{s} \\
& =O\left(\phi(m, \mu) \varepsilon_{k}\left(\frac{\alpha}{2}\right)\right)
\end{aligned}
$$

for any $\mu \in\left(0, \mu^{*}\right]$

Combining (A.16) with (A.17) and noting (A.15), we see that

$$
\begin{aligned}
& \mu\left\|\sum_{i=0}^{k} \Psi(k+1, i+1)\left[F_{i}-E\left(F_{i}\right)\right] \widetilde{\theta}_{i-m}\right\|_{2} \\
= & O\left([\sqrt{\mu} m+\phi(m, \mu)] \varepsilon_{k}\left(\frac{\alpha}{2}\right)\right)
\end{aligned}
$$


this in conjunction with (A.14) and (A.10) yields

$$
\begin{aligned}
& \left\|\widetilde{\theta}_{k+1}-\bar{\theta}_{k+1}\right\|_{2} \\
& =O\left([\sqrt{\mu} m+\phi(m, \mu)] \varepsilon_{k}\left(\frac{\alpha}{2}\right)\right)=O\left(\sigma(\mu) \varepsilon_{k}\left(\frac{\alpha}{2}\right)\right)
\end{aligned}
$$

Finally, substituting this and (A.5) into (A.3), we obtain $\forall \mu \in\left(0, \mu^{*}\right]$,

$$
\left\|E\left[\widetilde{\theta}_{k+1} \widetilde{\theta}_{k+1}^{\tau}\right]-\hat{\Pi}_{k+1}\right\| \leq O\left(\sigma(\mu)\left[\varepsilon_{k}\left(\frac{\alpha}{2}\right)\right]^{2}\right)
$$

Finally, substituting (A.4) into (A.18) we see that Theorem 4.1 is true.

\section{Appendix B: Analysis of the Riccati Equations}

(24) AND (25)

Lemma B.1. Let Condition P1) of Section 3 be satisfied and $\left\{S_{k}\right\}$ be bounded. Then the following two assertions hold:

(i) For $\left\{R_{k}\right\}$ recursively defined by (24) (with $R_{0}=P_{0}>$ 0 ), we have for all small $\mu>0$

$$
\left\|R_{k}-\bar{P}_{k}\right\|=O(\mu), \quad \forall k
$$

where $\left\{\bar{P}_{k}\right\}$ is defined recursively by $(45)$.

(ii) If $\left\{R_{k}\right\}$ is defined by (25) (with $R_{0}=P_{0} R^{-1}$ ) and $\left\{\bar{P}_{k}\right\}$ is defined by (54)), then for all small $\mu>0$,

$$
\left\|R_{k}-\bar{P}_{k} R^{-1}\right\|=O(\mu), \quad \forall k .
$$

Proof. (i) We first show that for all $m \geq 0$ and small $\mu>0$,

$$
R_{m h}>0, \quad \operatorname{tr}\left(R_{m h}\right)<2 d h \delta^{-1}+\operatorname{tr}\left(R_{0}\right) .
$$

where $\delta$ and $h$ are defined in $\mathrm{P} 1)$, and $d=\operatorname{dim}\left(\varphi_{k}\right)$.

Let (B.1) hold for some $m \geq 0$, we proceed to prove that (B.1) also holds with $m$ replaced by $m+1$. By (24), we know that $R_{k} \leq(1+\mu) R_{k-1}$, and that

$$
R_{k}=R_{k-1}^{1 / 2}\left[(1+\mu) I-\mu R_{k-1}^{1 / 2} S_{k} R_{k-1}^{1 / 2}\right] R_{k-1}^{1 / 2} .
$$

Consequently, it is easy to see that for all suitably small $\mu>0$

$$
R_{k}>0, \quad \forall k \in[m h,(m+1) h]
$$

Now, iterating (24) we see that $R_{(m+1) h}$ can be represented by $\left\{R_{m h}, S_{k}, m h+1 \leq k \leq(m+1) h\right\}$ as follows:

$$
R_{(m+1) h}=(1+\mu h) R_{m h}-\mu \sum_{k=m h+1}^{(m+1) h} R_{m h} S_{k} R_{m h}+\mu^{2} B_{m}
$$

where $\left\|B_{m}\right\| \leq B$ and $B$ depends only on $h$ and the upper bound of $\operatorname{tr}\left(R_{m h}\right)$ in (B.1).

Let us denote $r_{m} \stackrel{\delta}{=} \operatorname{tr}\left(R_{m h}\right)$. By the inequality $\operatorname{tr}\left(R_{m h}^{2}\right) \geq$ $d^{-1} r_{m}^{2}$ and the condition P1) we get from (B.3) that

$$
r_{m+1} \leq(1+\mu h) r_{m}-\mu \delta d^{-1} r_{m}^{2}+\mu^{2} B d .
$$

Note that the function $(1+\mu h) x-\mu \delta d^{-1} x^{2}$ is positive and increasing for $x \in\left(0, \frac{(1+\mu h) d}{2 \mu \delta}\right)$. Thus, if $\mu$ is small enough that

$$
M \stackrel{\delta}{=} 2 d h \delta^{-1}+\operatorname{tr}\left(R_{0}\right)<\frac{(1+\mu h) d}{2 \mu \delta} .
$$

Then by (B.4) and the assumption $r_{m} \leq M$, we have

$$
\begin{aligned}
r_{m+1} & \leq(1+\mu h) M-\mu \delta d^{-1} M^{2}+\mu^{2} B d . \\
& =M\left[1+\mu h-\mu \delta d^{-1} M+\mu^{2} B d M^{-1}\right] \\
& \leq M\left[1-\mu h+\mu^{2} B d M^{-1}\right] \\
& \leq M, \text { for small } \mu>0 .
\end{aligned}
$$

Hence by induction, (B.1) is proved. Consequently, (B.2) holds for all $m \geq 0$.

Furthermore, by (B.1) and the inequality

$$
R_{k} \leq(1+\mu) R_{k-1}
$$

it is easy to see that $\left\{R_{k}\right\}$ is uniformly bounded with respect to small $\mu>0$.

Next, by the matrix inversion formula from (24) we have

$R_{k}^{-1}=\frac{R_{k-1}^{-1}}{1+\mu}+\frac{\mu}{1+\mu} S_{k}^{1 / 2}\left[(1+\mu) I-\mu S_{k}^{1 / 2} R_{k-1} S_{k}^{1 / 2}\right]^{-1} S_{k}^{1 / 2}$

Since $\left\{R_{k}\right\}$ is bounded, from this it is evident that $\left\{R_{k}^{-1}\right\}$ is also bounded for small $\mu>0$, and that

$$
R_{k}^{-1}=(1-\mu) R_{k-1}^{-1}+\mu S_{k}+O\left(\mu^{2}\right) .
$$

This in conjunction with (45) gives

$$
\left\|R_{k}^{-1}-\bar{P}_{k}^{-1}\right\|=O(\mu)
$$

Hence, by the boundedness of $\left\{R_{k}\right\}$ and $\left\{\bar{P}_{k}\right\}$ (see, Lemma 5.2(i)), we get

$$
\left\|R_{k}-\bar{P}_{k}\right\|=\left\|R_{k}\left(\bar{P}_{k}^{-1}-R_{k}^{-1}\right) \bar{P}_{k}\right\|=O(\mu) .
$$

This proves the first assertion of the lemma

(ii) Similarly to the above analysis for (24), by (25) it can be shown that $\left\{R_{k}\right\}$ is a positive definite sequence and is uniformly bounded with respect to all small $\mu>0$.

Now, rewrite (25) as

$$
R_{k}=R_{k-1}^{1 / 2}\left(I-\mu R_{k-1}^{1 / 2} S_{k} R_{k-1}^{1 / 2}\right) R_{k-1}^{1 / 2}+\mu Q R^{-1}
$$

We see that

$$
\lambda_{\min }\left(R_{k}\right) \geq(1-\mu M) \lambda_{\min }\left(R_{k-1}\right)+\mu R^{-1} \lambda_{\min }(Q)
$$

where $M \stackrel{\delta}{=} \sup _{k}\left\|R_{k-1}^{1 / 2} S_{k} R_{k-1}^{1 / 2}\right\|$. This implies that $\left\{R_{k}^{-1}\right\}$ is also uniformly bounded with respect to all small $\mu>0$.

Note that (25) may also be rewritten as

$$
\begin{aligned}
R_{k} & =\left(I-\mu R_{k-1} S_{k}\right) R_{k-1}\left(I-\mu R_{k-1} S_{k}\right)^{\tau} \\
& +\mu R_{k-1} S_{k}^{1 / 2}\left(I-\mu S_{k}^{1 / 2} R_{k-1} S_{k}^{1 / 2}\right) S_{k}^{1 / 2} R_{k-1}+\mu Q R^{-1}
\end{aligned}
$$


Hence by applying Theorem 3.4 in [9] we know that $\left\{R_{k-1} S_{k}\right\} \in \mathcal{S}$, where $\mathcal{S}$ is defined by (29). Furthermore, by Proposition 4.1 we also know that $\left\{R_{k-1} S_{k}+O(\mu)\right\} \in \mathcal{S}$.

Next, by the matrix inversion formula it can be verified that

$$
R_{k}=\left(R_{k-1}^{-1}+\mu S_{k}\right)^{-1}+\mu R^{-1} Q+O\left(\mu^{2}\right) .
$$

Similarly to the proof of Lemma 5.5, from this and (54) we have

$$
\begin{aligned}
R_{k}- & \bar{P}_{k} R^{-1}=\left[I-\mu R_{k-1} S_{k}+O\left(\mu^{2}\right)\right] \\
\times & \times\left[R_{k-1}-\bar{P}_{k-1} R^{-1}\right]\left(I-\mu \bar{F}_{k}^{\tau}\right)+O\left(\mu^{2}\right)
\end{aligned}
$$

where $\left\{\bar{F}_{k}\right\}$ is defined as in Lemma 5.5. Since both $\left\{R_{k-1} S_{k}+O(\mu)\right\}$ and $\left\{\bar{F}_{k}\right\}$ belong to $\mathcal{S}$, by iterating the above equation it is easy to see that the desired result holds.

\section{REFERENCES}

[1] A. Benveniste. Design of adaptive algorithms for the tracking of time-varying systems. International Journal of Adaptive control and Signal Processing, 1:3-29, 1987.

[2] A. Benveniste, M. Metivier, and P. Priouret. Algorithmes adaptifs et approximations stochastiques. Masson, 1987.

[3] S. Bittanti and M. Campi. A fake information matrix approach to the analysis of finite memory RLS identification techniques. In IEEE Conference on Decision and Control, pages 2026-2031, San Antonio, TX, 1993.

[4] W. Gardner. Nonstationary learning characteristics of the LMS algorithms: A general study, analysis and critique. IEEE Trans. of Circuits and Systems, CAS-34(10):1199-1207, 1987.

[5] L. Gerencser. Almost sure exponential stability of random linear differential equations. Stochstics and Stochastics Reports, 36:91107, 1991.

[6] L. Gerencser. The law of the cubic root. In C. Banyasz and L. Keviczky, editors, Selected Papers from the Ninth IFAC/IFORS Symposium on System Identification, Budapest, Hungary, July 8-12, 1991.

[7] L. Guo. Estimating time-varying parameters by Kalman filter based algorithms: Stability and convergence. IEEE Trans. Autom. Control., AC-35:141-147, 1990.

[8] L. Guo. Stability of recursive stochastic tracking algorithms. SIAM Journal of Control and Optimization, 32:1195-1225, 1994.

[9] L. Guo and L. Ljung. Exponential stability of general tracking algorithms. IEEE Trans. Autom. Control, 1995. This Issue.

[10] L. Guo, L. Ljung, and P. Priouret. Performance analysis of the forgetting factor RLS algorithm. Int. J. Adaptive Control and Signal Processing, 7:525-537, 1993.

[11] P. Hall and C.C. Heyde. Martingale Limit Theory and Its Applications. Academic Press, New York, 1980.

[12] L. Ljung and S. Gunnarsson. Adaptive tracking in system identification - a survey. Automatica, 26(1):7-22, 1990.

[13] L. Ljung and P. Priouret. Remarks on the mean square tracking error. Int. J. of Adaptive Control, 5(6):395-403, 1991.

[14] L. Ljung and P. Priouret. A result of the mean square error obtained using general tracking algorithms. Int. J. of Adaptive Control, 5(4):231-250, 1991.

[15] L. Ljung and T. Söderström. Theory and Practice of Recursive Identification. MIT press, Cambridge, Mass., 1983.

[16] O. Macchi and E. Eweda. Second-order convergence analysis of stochastic adaptive linear filtering. IEEE Trans. Automatic Control, AC-28(1):76-85, 1983.

[17] M. Niedzwiecki and L. Guo. Nonasymptotic results for finite memory WLS filters. IEEE Trans. Automat. Control, AC36:198-206, 1991.

[18] R. Ravikanth and S.P. Meyn. Performance evaluation in identification and adaptive control of time varying systems. In Proc. of the 33rd IEEE Conference on Decision and Control, Lake Buena Vista, FL, 1994.

[19] R J Serfling. Contributions to central limit theory for dependent variables. The Annals of Math. Statistics, 39(4):1158-1175, 1968. Stability and Performance. Prentice Hall, 1994.

[21] B. Widrow and M. E. Hoff Jr. Adaptive switching circuits. IRE WESCON Convention Record, Part 4:96-104, 1960.

[22] B. Widrow and S. Stearns. Adaptive Signal Processing. PrenticeHall, Englewood-Cliffs, 1985. 